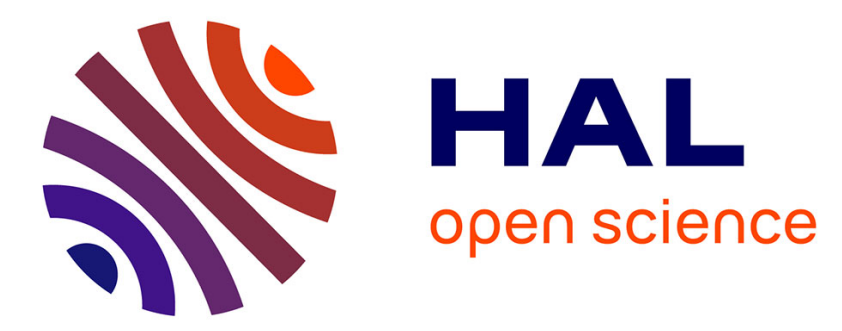

\title{
Internal Conversion and Intersystem Crossing with the Exact Factorization
}

\author{
Francesco Talotta, Sabine Morisset, Nathalie Rougeau, D. Lauvergnat, \\ Federica Agostini
}

\section{- To cite this version:}

Francesco Talotta, Sabine Morisset, Nathalie Rougeau, D. Lauvergnat, Federica Agostini. Internal Conversion and Intersystem Crossing with the Exact Factorization. Journal of Chemical Theory and Computation, 2020, 16 (8), pp.4833-4848. 10.1021/acs.jctc.0c00493 . hal-02994957

\section{HAL Id: hal-02994957 https://hal.science/hal-02994957}

Submitted on 10 Nov 2020

HAL is a multi-disciplinary open access archive for the deposit and dissemination of scientific research documents, whether they are published or not. The documents may come from teaching and research institutions in France or abroad, or from public or private research centers.
L'archive ouverte pluridisciplinaire HAL, est destinée au dépôt et à la diffusion de documents scientifiques de niveau recherche, publiés ou non, émanant des établissements d'enseignement et de recherche français ou étrangers, des laboratoires publics ou privés. 


\title{
Internal conversion and intersystem crossing with the exact factorization
}

\author{
Francesco Talotta, ${ }^{* \dagger, \uparrow}$ Sabine Morisset, ${ }^{\ddagger}$ Nathalie Rougeau, ${ }^{\ddagger}$ David Lauvergnat, ${ }^{\dagger}$ \\ and Federica Agostini*,† \\ $\dagger$ †niversité Paris-Saclay, CNRS, Institut de Chimie Physique UMR8000, 91405, Orsay, \\ France \\ $\ddagger$ Université Paris-Saclay, CNRS, Institut des Sciences Moléculaires d'Orsay, 91405, Orsay, \\ France \\ ๑Université Paris-Saclay, CNRS, Institut des Sciences Moléculaires d'Orsay, 91405, \\ Orsay, France \\ E-mail: francesco.talotta@universite-paris-saclay.fr; federica.agostini@universite-paris-saclay.fr
}

\begin{abstract}
We present a detailed derivation of the generalized coupled-trajectory mixed quantum-classical (G-CT-MQC) algorithm based on the exact factorization equations. The ultimate goal is to propose an algorithm that can be employed for molecular dynamics simulations of non-radiative phenomena, as the spin-allowed internal conversions and the spin-forbidden intersystem crossings. Internal conversions are nonadiabatic processes driven by the kinetic coupling between electronic states, whereas intersystem crossings are mediated by the spin-orbit coupling. In the paper we discuss computational issues related to the suitable representation for electronic dynamics and to the different nature of kinetic and spin-orbit coupling. Numerical studies on model systems allow us to test the performance of the G-CT-MQC algorithm in different situations.
\end{abstract}




\section{Introduction}

Trajectory-based molecular-dynamics methods are widely used to study processes in molecules that involve electronic excited states and their coupling. Photo-induced ultrafast phenomena triggered by the absorption of visible-UV light are an example: after the photoexcitation that populates electronic excited state(s), the molecule can relax to the ground state via radiationless channels such as the - spin-allowed - internal conversion, i.e., trough a path involving electronic states with the same spin multiplicity.1 Analogous ultrafast phenomena can be observed in systems with "large" spin-orbit coupling, 2 [1] where the nonradiative - spin-forbidden - process is then called intersystem crossing, if it involves states of different spin multiplicity that are (nearly) degenerate. Encountering intersystem crossings might seem likely only in the presence of heavy nuclei, since the spin-orbit coupling is a relativistic correction to the molecular Hamiltonian. However, this is not always the case, as spin-orbit coupling depends on the molecular geometry, and might affect the ultrafast non-radiative decay in photo-excited systems presenting light elements, $\frac{12}{20}$ in competition with other channels. Therefore, a trajectory-based scheme should be able to consistently treat internal conversions, mediated by the kinetic coupling between nuclear and electronic motion, and intersystem crossings, mediated by the spin-orbit coupling, in order to properly describe the molecular relaxation process.

In this paper, we will show how to treat kinetic nonadiabatic coupling (NAC), i.e., induced by nuclear motion, and spin-orbit coupling (SOC) in the framework of the exact factorization, 21,23 aiming to propose a trajectory-based generalized coupled-trajectory mixed quantum-classical (G-CT-MQC) algorithm $24 \sqrt[29]{20 r}$ simulating internal conversion (IC) and intersystem crossing (ISC) phenomena on equal footing. The algorithm has been presented in previous work,, 30 and validated on a model system. Here, we will (i) describe in detail the derivation of the algorithm starting from the exact-factorization equations, in order to present all the approximations the new algorithm is based on, (ii) analyze (numerically) exact results from quantum dynamics simulations on model systems using the perspective 
of the exact factorization to discuss those approximations, (iii) illustrate the performance of the new algorithm on additional examples.

Indeed, other trajectory-based approaches to ISC have been proposed previously. $\underline{31} \underline{39}$ Among the most successful and widely used we mention here ab initio multiple spawning (AIMS), $\underline{40 \mid 41}$ its generalized version, GAIMS, $\underline{31}$ and trajectory surface hopping (TSH). $\underline{32} \underline{34}$ In both cases, the existing schemes for IC have been adapted to include SOC: in GAIMS the spawning is mediated by the spin-orbit Hamiltonian; 31 in TSH the hopping procedure has been formulated either in the fewest-switches $\sqrt{33134139}$ or in the Landau-Zener ${ }^{32}$ fashion, in both cases induced, as in GAIMS, by the matrix elements of the spin-orbit Hamiltonian. In this context, critical issues have been addressed, $\underline{31+3439}$ that will be as well the focus of our discussions in this work, and they are (i) the electronic representation used to describe the electronic sub-system, i.e., the spin-diabatic and spin-adiabatic representations, and (ii) the delocalized nature of SOC in nuclear space, that is fundamentally different from the (usually) localized NAC. It is important to mention here, that other strategies, rather than trajectory-based schemes, have been developed to simulate ISCs, as wavepacket propagation techniques. $\frac{12[17 / 42 / 43}{2}$

The presentation of our results is organized as follows. First, we formulate the exact

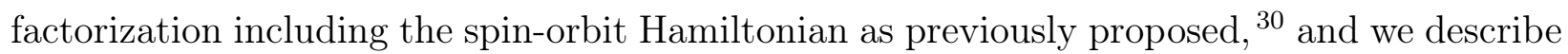
in detail the approximations leading to inclusion of SOC in the CT-MQC algorithm (G-CTMQC). Then, based on an illustrative one-singlet/one-triplet model ${ }^{30 \mid 39}$ we analyze (numerically) exact quantum dynamics to discuss the validity of the approximations which G-CTMQC is based on. Comparison between quantum-dynamics results and the trajectory-based results is presented for the one-singlet/one-triplet model, and for a two-singlet/one-triplet model. $\stackrel{39}{ }$ Conclusions and perspectives are presented in the closing section. 


\section{Nonadiabatic and spin-orbit coupling with the exact fac- torization}

A system of interacting electrons and nuclei, considering spin-orbit corrections to the nonrelativistic molecular Hamiltonian, is described by

$$
\hat{H}(\mathbf{x}, \mathbf{R})=\hat{H}_{m o l}(\mathbf{x}, \mathbf{R})+\hat{H}_{S O}(\mathbf{x}, \mathbf{R}),
$$

with electronic position-spin variables labelled as $\mathbf{x}=[\mathbf{r}, \boldsymbol{\sigma}]$, and nuclear positions labelled as R. We define the "molecular Hamiltonian" as the sum of the nuclear kinetic energy and the electronic Hamiltonian, i.e., $\hat{H}_{m o l}(\mathbf{x}, \mathbf{R})=\hat{T}_{n}+\hat{H}_{e l}(\mathbf{x}, \mathbf{R})$, whereas $\hat{H}_{S O}(\mathbf{x}, \mathbf{R})$ is the spin-

orbit coupling. Note that, $\hat{H}_{m o l}(\mathbf{x}, \mathbf{R})$ is block-diagonal in spin space, $\hat{H}_{e l}(\mathbf{x}, \mathbf{R})$ contains the sum of the electronic kinetic energy and the interaction potentials, and the particular form ${ }^{3214445}$ chosen for $\hat{H}_{S O}(\mathbf{x}, \mathbf{R})$ does not affect any of the results presented below.

The time-dependent Schrödinger equation with Hamiltonian (1) is

$$
i \hbar \partial_{t} \Psi(\mathbf{x}, \mathbf{R}, t)=\hat{H} \Psi(\mathbf{x}, \mathbf{R}, t),
$$

whose solution, $\Psi(\mathbf{x}, \mathbf{R}, t)$, is written as the product of a nuclear wavefunction, $\chi(\mathbf{R}, t)$, and an electronic conditional factor, $\Phi_{\mathbf{R}}(\mathbf{x}, t)$, that parametrically depends on $\mathbf{R}$, namely,

$$
\Psi(\mathbf{x}, \mathbf{R}, t)=\chi(\mathbf{R}, t) \Phi_{\mathbf{R}}(\mathbf{x}, t) .
$$

To guarantee that $|\chi(\mathbf{R}, t)|^{2}$ reproduces at all times the (exact) nuclear density obtained from $\Psi(\mathbf{x}, \mathbf{R}, t)$, the partial normalization condition (PNC)

$$
\int d \mathbf{x}\left|\Phi_{\mathbf{R}}(\mathbf{x}, t)\right|^{2}=\sum_{\sigma_{1}} \sum_{\sigma_{2}} \ldots \int d \mathbf{r}_{1} \int d \mathbf{r}_{2} \ldots\left|\Phi_{\mathbf{R}}\left(\mathbf{r}_{1}, \sigma_{1}, \mathbf{r}_{2}, \sigma_{2}, \ldots, t\right)\right|^{2}=1 \quad \forall \mathbf{R}, t
$$


is imposed. Here, we have $N_{e l}$ sums over the spins of the electrons and $N_{e l} 3$-dimensional integrals over the positions of the electrons.

After having introduced the factored form of the electron-nuclear wavefunction, Eq. (3), and the PNC, Eq. (44), it is easy to show with some algebra, ${ }^{22146}$ 年 as done for the original derivation of the exact-factorization equations, that the evolution of $\chi(\mathbf{R}, t)$ and $\Phi_{\mathbf{R}}(\mathbf{x}, t)$ is given by

$$
\begin{aligned}
i \hbar \partial_{t} \chi(\mathbf{R}, t) & =\left[\sum_{\nu=1}^{N_{n}} \frac{\left[-i \hbar \nabla_{\nu}+\mathbf{A}_{\nu}(\mathbf{R}, t)\right]^{2}}{2 M_{\nu}}+\epsilon(\mathbf{R}, t)\right] \chi(\mathbf{R}, t) \\
i \hbar \partial_{t} \Phi_{\mathbf{R}}(\mathbf{x}, t) & =\left[\hat{H}_{e l}(\mathbf{x}, \mathbf{R})+\hat{H}_{S O}(\mathbf{x}, \mathbf{R})+\hat{U}_{e n}\left[\Phi_{\mathbf{R}}, \chi\right]-\epsilon(\mathbf{R}, t)\right] \Phi_{\mathbf{R}}(\mathbf{x}, t) .
\end{aligned}
$$

The form of the nuclear kinetic energy is $\hat{T}_{n}=-\sum_{\nu} \hbar^{2} \nabla_{\nu}^{2} /\left(2 M_{\nu}\right)$, where the symbol $\nabla_{\nu}$ indicates the gradient taken with respect to the position of the nucleus $\nu$ (with mass $M_{\nu}$ ). The time-dependent potentials of the exact factorization mediate the coupling between electrons and nuclei, beyond the adiabatic regime, and they are: the time-dependent vector potential ${ }^{49}[52$

$$
\mathbf{A}_{\nu}(\mathbf{R}, t)=\left\langle\Phi_{\mathbf{R}}(t) \mid-i \hbar \nabla_{\nu} \Phi_{\mathbf{R}}(t)\right\rangle_{\mathbf{x}}
$$

and the time-dependent scalar potential, or time-dependent potential energy surface (TD$\mathrm{PES}) \longdiv { 5 3 \lcm { 5 8 } }$

$$
\epsilon(\mathbf{R}, t)=\left\langle\Phi_{\mathbf{R}}(t)\left|\hat{H}_{e l}(\mathbf{x}, \mathbf{R})+\hat{H}_{S O}(\mathbf{x}, \mathbf{R})+\hat{U}_{e n}\left[\Phi_{\mathbf{R}}, \chi\right]-i \hbar \partial_{t}\right| \Phi_{\mathbf{R}}(t)\right\rangle_{\mathbf{x}} .
$$

We have indicated the integration over $\mathbf{x}$ as $\langle\cdot\rangle_{\mathbf{x}}$. The electron-nuclear coupling operator 


$$
\begin{aligned}
& \hat{U}_{e n}\left[\Phi_{\mathbf{R}}, \chi\right] \text { is }{ }^{59}[1] \\
& \hat{U}_{e n}\left[\Phi_{\mathbf{R}}, \chi\right]=\sum_{\nu=1}^{N_{n}} \frac{1}{M_{\nu}}\left[\frac{\left[-i \hbar \nabla_{\nu}+\mathbf{A}_{\nu}(\mathbf{R}, t)\right]^{2}}{2}+\left(\frac{-i \hbar \nabla_{\nu} \chi(\mathbf{R}, t)}{\chi(\mathbf{R}, t)}+\mathbf{A}_{\nu}(\mathbf{R}, t)\right) \cdot\left(-i \hbar \nabla_{\nu}-\mathbf{A}_{\nu}(\mathbf{R}, t)\right)\right]
\end{aligned}
$$

and depends explicitly on the nuclear wavfunction $\chi(\mathbf{R}, t)$, and implicitly on the electronic factor $\Phi_{\mathbf{R}}(\mathbf{r}, t)$, via its dependence on the vector potential.

It is clear from the above discussion, that, formally, the inclusion of the spin-orbit Hamiltonian does not alter the features of the exact factorization: the expression of the timedependent vector potential is unaffected, while the SOC appears in the definition of the TDPES. Moreover, the gauge freedom (the only freedom in Eq. (3)) is still expressed by the multiplication of each term of Eq. (3) by a $(\mathbf{R}, t)$-dependent phase factor, that leaves the product unchanged. Thus, an additional equation to fix the gauge has to be introduced to guarantee uniqueness of the solution of the evolution equations.

As discussed in the Introduction, the electronic wavefunction can be represented either in the spin-diabatic basis,

$$
\hat{H}_{e l}(\mathbf{x}, \mathbf{R}) \varphi_{\mathbf{R}}^{\left(S_{J}, M_{S_{J}}\right)}(\mathbf{x})=\epsilon_{s d}^{\left(S_{J}, M_{S_{J}}\right)}(\mathbf{R}) \varphi_{\mathbf{R}}^{\left(S_{J}, M_{S_{J}}\right)}(\mathbf{x})
$$

formed by the eigenstates of electronic Hamiltonian (without SOC), or in the spin-adiabatic basis,

$$
\left[\hat{H}_{e l}(\mathbf{x}, \mathbf{R})+\hat{H}_{S O}(\mathbf{x}, \mathbf{R})\right] \varphi_{\mathbf{R}}^{(j)}(\mathbf{x})=\epsilon_{s a}^{(j)}(\mathbf{R}) \varphi_{\mathbf{R}}^{(j)}(\mathbf{x})
$$

formed by the eigenstates of the electronic Hamiltonian with SOC. The spin-diabatic states of Eq. 10 are eigenstates of $\hat{S}^{2}$ and $\hat{S}_{z}$, with eigenvalues $\hbar^{2} S_{J}\left(S_{J}+1\right)$ and $\hbar M_{S_{J}}$, respectively. The index $S_{J}$ labels the multiplets, each including $2 S_{J}+1$ states corresponding to the eigenvalues $\epsilon_{s d}^{\left(S_{J}, M_{S_{J}}\right)}(\mathbf{R})$. In Eq. 11 the index $j$ labels the spin-adiabatic states, which are 
combinations of different spin multiplicities, whose eigenvalues are indicated as $\epsilon_{s a}^{(j)}(\mathbf{R})$. To simplify the notation in the following equations, we will use only the index $J$ to label the spin-multiplets and the components of each multiplet, such that for instance $\sum_{J}$ stands for $\sum_{S_{J}} \sum_{M_{S_{J}}=-S_{J}}^{M_{S_{J}}=+S_{J}}$

The electronic wavefunciton $\Phi_{\mathbf{R}}(\mathbf{x}, t)$ can be expressed as the linear combinations

$$
\begin{aligned}
\Phi_{\mathbf{R}}(\mathbf{x}, t) & =\sum_{J} C_{J}^{s d}(\mathbf{R}, t) \varphi_{\mathbf{R}}^{(J)}(\mathbf{x}) \\
& =\sum_{j} C_{j}^{s a}(\mathbf{R}, t) \varphi_{\mathbf{R}}^{(j)}(\mathbf{x})
\end{aligned}
$$

with the normalization conditions $\sum_{J}\left|C_{J}^{s d}(\mathbf{R}, t)\right|^{2}=\sum_{j}\left|C_{j}^{s a}(\mathbf{R}, t)\right|^{2}=1 \forall \mathbf{R}, t$ that follow from the PNC of Eq. (4) and from the orthonormality of the electronic states. We will refer in the following to $C_{J}^{s d}(\mathbf{R}, t)$ and $C_{j}^{s a}(\mathbf{R}, t)$ as the spin-diabatic and spin-adiabatic electronic coefficients, respectively.

Note that also the full electron-nuclear wavefunction $\Psi(\mathbf{x}, \mathbf{R}, t)$ can be expressed in the two basis, as

$$
\begin{aligned}
\Psi(\mathbf{x}, \mathbf{R}, t) & =\sum_{J} \chi_{J}^{s d}(\mathbf{R}, t) \varphi_{\mathbf{R}}^{(J)}(\mathbf{x}) \\
& =\sum_{j} \chi_{j}^{s a}(\mathbf{R}, t) \varphi_{\mathbf{R}}^{(j)}(\mathbf{x})
\end{aligned}
$$

where the relations $\chi_{J}^{s d}(\mathbf{R}, t)=\chi(\mathbf{R}, t) C_{J}^{s d}(\mathbf{R}, t)$ and $\chi_{j}^{s a}(\mathbf{R}, t)=\chi(\mathbf{R}, t) C_{j}^{s a}(\mathbf{R}, t)$ follow from Eq. (3). The functions $\chi_{J}^{s d}(\mathbf{R}, t)$ and $\chi_{j}^{s a}(\mathbf{R}, t)$ can be referred to as spin-diabatic and spin-adiabatic nuclear amplitudes, and have the property

$$
|\chi(\mathbf{R}, t)|^{2}=\sum_{J}\left|\chi_{J}^{s d}(\mathbf{R}, t)\right|^{2}=\sum_{j}\left|\chi_{j}^{s a}(\mathbf{R}, t)\right|^{2}
$$

that can be derived using the PNC of Eq. (4) in the two basis.

Inserting either Eq. (12) or Eq. (13) into the electronic evolution equation (6) allows to 
derive coupled evolution equations for the electronic coefficients. Clearly, exact integration in either basis would yield the same result, with the same time-dependent vector potential and TDPES (this property will be proven numerically later on), provided both basis span the same subspace. Moreover, since nuclear dynamics is driven by these two time-dependent potentials, the nuclear evolution as well would be not affected by the use of different electronic basis. This feature is what makes the exact factorization a suitable working framework to construct a quantum-classical algorithm for simulations of IC and ISC processes, where nuclear classical-like trajectories are generated by forces from the vector potential and the TDPES: the exact-factorization equations do not depend on the chosen representation for the electronic sub-system. Nonetheless, approximations are needed to be able to solve Eqs. (5) and (6), not only for "real" systems ${ }^{26}$ but even for simple models. $\frac{48 \mid 62}{62}$ The approximations that will be introduced in the electronic equation (6) will be the cause of differences in performance of the quantum-classical algorithm in the spin-diabatic and in the spin-adiabatic basis, as we will carefully discuss below.

The use of the spin-diabatic representation is particularly suitable for on-the-fly simulations based on the interface between trajectory-based nuclear dynamics and quantumchemistry calculations to evaluate electronic-structure properties along the trajectories. As far as we know, standard quantum-chemistry softwares do not provide nuclear gradients of the SOC, thus, classical forces are only computed for the spin-diabatic energy eigenvalues of Eq. $10, \cdot 32+34$ In the development of a new algorithm that includes effects due to SOC, it is necessary to keep in mind that the algorithm should be formulated in the spindiabatic representation, allowing flexibility in the level of electronic-structure theory accessible. However, semi-empirical electronic-structure approaches, where nuclear gradients of SOC are computed, are currently available,, 63 therefore formulation of the new algorithm in the spin-adiabatic representation as well is desirable. 


\section{Trajectory-based description}

The nuclear wavefunction is written as $\chi(\mathbf{R}, t)=|\chi(\mathbf{R}, t)| \exp [(i / \hbar) S(\mathbf{R}, t)]$, i.e., in polar representation, in terms of its modulus and phase, such that the coupled evolution equations

$$
\begin{aligned}
-\partial_{t} S(\mathbf{R}, t) & =\sum_{\nu} \frac{\left[\nabla_{\nu} S(\mathbf{R}, t)+\mathbf{A}_{\nu}(\mathbf{R}, t)\right]^{2}}{2 M_{\nu}}+\epsilon(\mathbf{R}, t) \\
\partial_{t}|\chi(\mathbf{R}, t)|^{2} & =-\sum_{\nu} \nabla_{\nu} \cdot\left[\frac{\nabla_{\nu} S(\mathbf{R}, t)+\mathbf{A}_{\nu}(\mathbf{R}, t)}{M_{\nu}}|\chi(\mathbf{R}, t)|^{2}\right]
\end{aligned}
$$

are derived from the nuclear time-dependent Schrödinger equation (5). Here, we give the equation for the phase $S(\mathbf{R}, t)$ in the classical limit, that is by neglecting in Eq. (17) the quantum potential term, i.e., $-\hbar^{2} \sum_{\nu}\left(\nabla_{\nu}^{2}|\chi(\mathbf{R}, t)|\right) /\left(2 M_{\nu}|\chi(\mathbf{R}, t)|\right)$. The first equation can be solved with the method of characteristics, introducing a set of ordinary differential equations - the characteristic equations - yielding the values of the field $S(\mathbf{R}, t) \forall \mathbf{R}, t$. The

characteristic equations are Hamilton-like equations that describe the evolution in time of the "variables" $\mathbf{R}_{\nu}(t)$ and $\mathbf{P}_{\nu}(t) \equiv \nabla_{\nu} S(\mathbf{R}(t), t)+\mathbf{A}_{\nu}(\mathbf{R}(t), t)$ appearing in Eq. (17), i.e.,

$$
\begin{aligned}
\dot{\mathbf{R}}_{\nu}(t) & =\frac{\mathbf{P}_{\nu}(t)}{M_{\nu}} \\
\dot{\mathbf{P}}_{\nu}(t) & =-\nabla_{\nu}\left(\epsilon(\mathbf{R}(t), t)+\sum_{\nu^{\prime}} \dot{\mathbf{R}}_{\nu^{\prime}}(t) \cdot \mathbf{A}_{\nu^{\prime}}(\mathbf{R}(t), t)\right)+\dot{\mathbf{A}}_{\nu}(\mathbf{R}(t), t) .
\end{aligned}
$$

The procedure to derive Eqs. (19) and (20) has been illustrated in detail in Ref., $\frac{48}{\text { therefore, }}$ we just mention here that the first term on the right-hand side of Eq. (20) is the gradient of the (pseudo-classical) Hamiltonian defined on the right-hand side of Eq. (17). Furthermore, it is worth pointing out that the time-dependent potentials $\epsilon(\mathbf{R}(t), t)$ and $\mathbf{A}_{\nu}(\mathbf{R}(t), t)$ are evaluated along each characteristic $\mathbf{R}(t)$ by solving the electronic equation (6) along the same characteristic. Solving the partial differential equation (6) along the flow of trajectories the characteristics - requires to switch from the Eulerian frame to the Lagrangian frame (see Eq. (22) below), but before discussing the electronic equation, let us discuss the continuity 
equation (18).

The evolution of the nuclear density, Eq. (18), is described by a "standard" continuity equation, that can be solved coupled to Eqs. (19) and (20). This would allow to reconstruct the nuclear wavefunction, even though not exactly because we have already dropped the quantum potential in Eq. (17). Neglecting the quantum potential has the effect of decoupling the evolution of the phase from the evolution of the density, while the opposite is not true. Therefore, as done in previous work, $\stackrel{25 / 48}{2}$ we will not solve the continuity equation, and we will only reconstruct a classical-like nuclear density from the distribution of the trajectories, working in the hypothesis that for short enough times, an ensemble of trajectories evolving with Eqs. 19 and $(20)$ will sample portions of nuclear configuration space with high probability density.

Eqs. (19) and (20) are the basis of the CT-MQC algorithm, that has been derived and tested in Refs., ${ }^{2425}$ applied in Refs. ${ }^{26127}$ in combination with time-dependent density functional theory to study the photo-induced dynamics in oxirane, and thoroughly analyzed in Refs. $\frac{28164}{26}$

The electronic equation (6) is expressed along the characteristics $\mathbf{R}(t)$ as

$$
\begin{aligned}
& \dot{\Phi}_{\mathbf{R}(t)}(\mathbf{x}, t)-\sum_{\nu} \dot{\mathbf{R}}_{\nu}(t) \cdot \nabla_{\nu} \Phi_{\mathbf{R}(t)}(\mathbf{x}, t)=-\frac{i}{\hbar} {\left[\hat{H}_{e l}(\mathbf{x}, \mathbf{R}(t))+\hat{H}_{S O}(\mathbf{x}, \mathbf{R}(t))\right.} \\
&\left.+\hat{U}_{e n}\left[\Phi_{\mathbf{R}(t)}, \chi\right]-\epsilon(\mathbf{R}(t), t)\right] \Phi_{\mathbf{R}(t)}(\mathbf{x}, t) .
\end{aligned}
$$

Switching from the Eulerian to the Lagrangian frame, only total time derivatives can be evaluated along the flow, that is why the symbol $\dot{\Phi}_{\mathbf{R}(t)}(\mathbf{x}, t)$ has been introduced. Furthermore, we used the relation

$$
\dot{\Phi}_{\mathbf{R}(t)}(\mathbf{x}, t)=\frac{\partial \Phi_{\mathbf{R}(t)}(\mathbf{x}, t)}{\partial t}+\sum_{\nu} \dot{\mathbf{R}}_{\nu}(t) \cdot \nabla_{\nu} \Phi_{\mathbf{R}(t)}(\mathbf{x}, t)
$$

to replace the partial time derivative of Eq. (6) with the total derivative, and thus derive the 
term on the left-hand side of Eq. (21). From Eq. (21) it is clear that the "new" definition of the TDPES along the trajectories is

$$
\begin{aligned}
\epsilon(\mathbf{R}(t), t)= & \left\langle\Phi_{\mathbf{R}(t)}(t)\left|\hat{H}_{e l}(\mathbf{R}(t))+\hat{H}_{S O}(\mathbf{R}(t))+\hat{U}_{e n}\left[\Phi_{\mathbf{R}(t)}, \chi\right]\right| \Phi_{\mathbf{R}(t)}(t)\right\rangle_{\mathbf{x}} \\
& -i \hbar\left\langle\Phi_{\mathbf{R}(t)}(t) \mid \dot{\Phi}_{\mathbf{R}(t)}(t)\right\rangle_{\mathbf{x}}-\sum_{\nu} \dot{\mathbf{R}}_{\nu}(t) \cdot \mathbf{A}_{\nu}(\mathbf{R}(t), t)
\end{aligned}
$$

obtained by multiplying Eq. (21) on the left by $\Phi_{\mathbf{R}(t)}(\mathbf{x}, t)$ and integrating over $\mathbf{x}$, and using Eq. (4) $\forall \mathbf{R}(t), t$. Note that the second term on the left-hand side of Eq. (21) yields the vector potential in Eq. (23).

From this point on, we will use the expressions of $\Phi_{\mathbf{R}(t)}(\mathbf{x}, t)$ in the spin-diabatic and in the spin-adiabatic basis, thus the equations for the electronic coefficients will look slightly different. We will first present in detail the derivation of the equations in the spin-diabatic representation, then we will briefly report the equations in the spin-adiabatic representation because they follow from the original derivation of CT-MQC.

\section{Electronic dynamics: Spin-diabatic representation}

We use the expression of $\Phi_{\mathbf{R}(t)}(\mathbf{x}, t)$ in the spin-diabatic basis, given in Eq. (12), noting that the electronic coefficients and the states acquire a dependence on the trajectory, namely

$$
\Phi_{\mathbf{R}(t)}(\mathbf{x}, t)=\sum_{J} C_{J}^{s d}(\mathbf{R}(t), t) \varphi_{\mathbf{R}(t)}^{(J)}(\mathbf{x})
$$


We insert this expression into the evolution equation 21 , then projecting onto $\varphi_{\mathbf{R}(t)}^{(K)}(\mathbf{x})$, we find

$$
\begin{aligned}
\dot{C}_{K}^{s d}(\mathbf{R}(t), t)= & -\frac{i}{\hbar}\left[\epsilon_{s d}^{(K)}(\mathbf{R}(t), t)-\epsilon(\mathbf{R}(t), t)\right] C_{K}^{s d}(\mathbf{R}(t), t) \\
& -\frac{i}{\hbar} \sum_{J}\left\langle\varphi_{\mathbf{R}(t)}^{(K)}\left|\left[\hat{H}_{S O}(\mathbf{R}(t))+\hat{U}_{e n}\right] C_{J}^{s d}(\mathbf{R}(t), t)\right| \varphi_{\mathbf{R}(t)}^{(J)}\right\rangle_{\mathbf{x}} \\
& +\sum_{\nu} \dot{\mathbf{R}}_{\nu}(t) \cdot \nabla_{\nu} C_{K}^{s d}(\mathbf{R}(t), t) .
\end{aligned}
$$

When using the expansion (24) in the left-hand side of Eq. (21), we have

$$
\dot{\Phi}_{\mathbf{R}(t)}(\mathbf{x}, t)=\sum_{J} \dot{C}_{J}^{s d}(\mathbf{R}(t), t) \varphi_{\mathbf{R}(t)}^{(J)}(\mathbf{x})+C_{J}^{s d}(\mathbf{R}(t), t) \dot{\varphi}_{\mathbf{R}(t)}^{(J)}(\mathbf{x})
$$

and applying in the second term on the right-hand side the total time derivative to the diabatic state similarly to Eq. (22), we find that the partial time derivative is zero because $\varphi_{\mathbf{R}(t)}^{(J)}(\mathbf{x})$ depends on time only via its dependence on the trajectory, then

$$
\dot{\Phi}_{\mathbf{R}(t)}(\mathbf{x}, t)=\sum_{J} \dot{C}_{J}^{s d}(\mathbf{R}(t), t) \varphi_{\mathbf{R}(t)}^{(J)}(\mathbf{x})+\sum_{J} \sum_{\nu} C_{J}^{s d}(\mathbf{R}(t), t) \dot{\mathbf{R}}_{\nu}(t) \cdot \nabla_{\nu} \varphi_{\mathbf{R}(t)}^{(J)}(\mathbf{x}) .
$$

Let us now provide an explicit expression for the term in Eq. 25b that contains the electronnuclear coupling operator $\hat{U}_{e n}$. In the definition $(9)$ of $\hat{U}_{e n}$ we identify two terms: the first term has been shown ${ }^{65}$ to be smaller if compared to the second term, and we will thus neglect it henceforth. In addition, such term contains second-order spatial derivatives of the spin-diabatic states, which are usually not available in quantum-chemistry codes to compute electronic-structure properties. The corresponding term in Eq. (23) will be neglected as well to maintain gauge invariance of the exact-factorization equations in their trajectory-based 


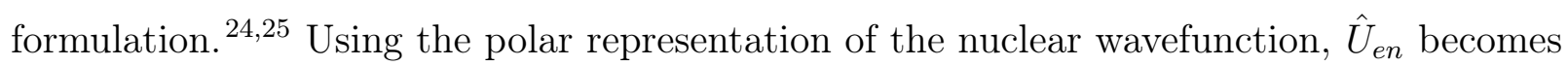

$$
\begin{aligned}
\hat{U}_{e n} & \simeq \sum_{\nu}\left(\frac{\nabla_{\nu} S(\mathbf{R}(t), t)+\mathbf{A}_{\nu}(\mathbf{R}(t), t)}{M_{\nu}}+i \frac{-\hbar \nabla_{\nu}|\chi(\mathbf{R}(t), t)|}{M_{\nu}|\chi(\mathbf{R}(t), t)|}\right) \cdot\left(-i \hbar \nabla_{\nu}-\mathbf{A}_{\nu}(\mathbf{R}(t), t)\right) \\
& =\sum_{\nu}\left[\dot{\mathbf{R}}_{\nu}(t)+i \frac{\mathcal{Q}_{\nu}(\mathbf{R}(t), t)}{M_{\nu}}\right] \cdot\left(-i \hbar \nabla_{\nu}-\mathbf{A}_{\nu}(\mathbf{R}(t), t)\right)
\end{aligned}
$$

The first term in parenthesis of Eq. $28 \mathrm{a}$ is the velocity of the trajectory, $\dot{\mathbf{R}}_{\nu}(t)$ (from the characteristic equation (19p), whereas the second term contains the quantum momentum, 24 $\mathcal{Q}_{\nu}(\mathbf{R}(t), t)$, that has been defined in previous work on CT-MQC. The quantum momentum induces quantum decoherence effects by tracking the spatial delocalization over time of the nuclear density (or, equivalently, of its modulus). 28164

Using Eq. (28b) in the expression of $\dot{C}_{K}^{s d}(\mathbf{R}(t), t)$, we finally derive

$$
\begin{aligned}
\dot{C}_{K}^{s d}(\mathbf{R}(t), t)= & -\frac{i}{\hbar}\left[\epsilon_{s d}^{(K)}(\mathbf{R}(t), t)-\epsilon(\mathbf{R}(t), t)\right] C_{K}^{s d}(\mathbf{R}(t), t) \\
& -\frac{i}{\hbar} \sum_{J}\left[\hat{H}_{S O}(\mathbf{R}(t))\right]_{K J} C_{J}^{s d}(\mathbf{R}(t), t) \\
& +\frac{i}{\hbar} \sum_{\nu}\left[\dot{\mathbf{R}}_{\nu}(t)+i \frac{\mathcal{Q}_{\nu}(\mathbf{R}(t), t)}{M_{\nu}}\right] \cdot \mathbf{A}_{\nu}(\mathbf{R}(t), t) C_{K}^{s d}(\mathbf{R}(t), t) \\
& -\sum_{\nu} i \frac{\mathcal{Q}_{\nu}(\mathbf{R}(t), t)}{M_{\nu}} \cdot \nabla_{\nu} C_{K}^{s d}(\mathbf{R}(t), t) \\
& -\sum_{J} \sum_{\nu}\left[\dot{\mathbf{R}}_{\nu}(t)+i \frac{\mathcal{Q}_{\nu}(\mathbf{R}(t), t)}{M_{\nu}}\right] \cdot \mathbf{d}_{\nu ; K J}(\mathbf{R}(t)) C_{J}^{s d}(\mathbf{R}(t), t),
\end{aligned}
$$

where we introduced a new symbol to indicate the NAC vector in spin-diabatic basis, namely

$$
\mathbf{d}_{\nu ; K J}(\mathbf{R}(t))=\left\langle\varphi_{\mathbf{R}(t)}^{(K)} \mid \nabla_{\nu} \varphi_{\mathbf{R}(t)}^{(J)}\right\rangle_{\mathbf{x}}
$$

Equations 29 give the evolution of the spin-diabatic electronic coefficients along the trajectory $\mathbf{R}(t)$; it is an approximate form of Eqs. (25) because of the neglect of the first term 
on the right-hand side of the definition (9) of the electron-nuclear coupling operator. We can identify three "diagonal" terms, i.e., Eqs. 29a), 29c and (29d), and two "off-diagonal" terms expressing the coupling between the spin-diabatic states $K$ and $J$ mediated by the SOC and the NAC, i.e., Eqs. (29b) and (29e), respectively.

As proposed in previous work, $\stackrel{25128}{2}$ we set the gauge such that

$$
\epsilon(\mathbf{R}(t), t)+\sum_{\nu} \dot{\mathbf{R}}_{\nu}(t) \cdot \mathbf{A}_{\nu}(\mathbf{R}(t), t)=0
$$

and introduce the following approximations:

(i) neglect the term containing scalar product of $\mathcal{Q}_{\nu}(\mathbf{R}(t), t)$ and $\mathbf{d}_{\nu ; K J}(\mathbf{R}(t))$, working in the hypothesis that decoherence effects start playing a role after the action of NAC;

(ii) neglect the spatial derivative of the modulus of the electronic coefficients $\left|C_{K}^{s d}(\mathbf{R}(t), t)\right|$, while keeping the spatial derivative of their phase $\gamma_{K}^{s d}(\mathbf{R}(t), t)$, such that

$$
\begin{aligned}
\nabla_{\nu} C_{K}^{s d}(\mathbf{R}(t), t) & =\nabla_{\nu}\left|C_{K}^{s d}(\mathbf{R}(t), t)\right| \exp \left[\frac{i}{\hbar} \gamma_{K}^{s d}(\mathbf{R}(t), t)\right] \\
& \simeq \frac{i}{\hbar}\left(\nabla_{\nu} \gamma_{K}^{s d}(\mathbf{R}(t), t)\right) C_{K}^{s d}(\mathbf{R}(t), t) \\
& =\frac{i}{\hbar} \mathbf{f}_{\nu ; K}^{s d}(\mathbf{R}(t)) C_{K}^{s d}(\mathbf{R}(t), t),
\end{aligned}
$$

(iii) the gradient of the phase of the electronic coefficients, denoted $\mathbf{f}_{\nu ; K}^{s d}(\mathbf{R}(t))$, is approximated as

$$
\mathbf{f}_{\nu ; K}^{s d}(\mathbf{R}(t))=\int^{t} d \tau\left(-\nabla_{\nu} \epsilon_{s d}^{(K)}(\mathbf{R}(t))\right)
$$

that is the spin-diabatic force for state $K$ accumulated over time along the trajectory. The Appendix provides details on the derivation of this expression.

The gauge condition imposed above simplifies the characteristic equation 20, reducing it to $\dot{\mathbf{P}}_{\nu}(t)=\dot{\mathbf{A}}_{\nu}(t)$. Note that with the choice of gauge made in Eq. 31] we circumvent calculations of gradients of the SOC matrix elements appearing in the expression of the 
TDPES (according to Eq. 23), in fact, $\hat{H}_{S O}$ appears in the definition of the TDPES).

While the neglect of the term containing the scalar product of the quantum momentum and the NAC vectors, that is approximation (i) above, could be easily relaxed, approximations (ii) and (iii) are crucial to avoid the calculations of spatial derivatives of the electronic coefficients. As indicated by the symbol $C_{K}^{s d}(\mathbf{R}(t), t)$, the value of the electronic coefficient is only known at the position of the trajectory; calculations of spatial derivatives rely on the distribution of the trajectories in nuclear configuration space, that is usually noisy (not regular) and, thus, source of numerical errors. In addition, note that approximation (ii) has been introduced in the original derivation of CT-MQC based on the analysis of the exact factorization in the adiabatic representation. $\frac{535456}{54}$ Therefore, the "performance" of this approximation should be expected to be different in the spin-diabatic and in the spin-adiabatic representations, as we will discuss below.

The final expression used in G-CT-MQC for the evolution of the spin-diabatic coefficients $\dot{C}_{K}^{s d}(\mathbf{R}(t), t)$, putting together the choice of the gauge with approximations (i), (ii) and (iii) is

$$
\begin{aligned}
\dot{C}_{K}^{s d}(t)= & {\left[-\frac{i}{\hbar} \epsilon_{s d}^{(K)}(t)+\sum_{\nu} \frac{\mathcal{Q}_{\nu}(t)}{\hbar M_{\nu}} \cdot\left(\mathbf{f}_{\nu ; K}^{s d}(t)-\mathbf{A}_{\nu}(t)\right)\right] C_{K}^{s d}(t) } \\
& -\sum_{J}\left(\frac{i}{\hbar}\left[\hat{H}_{S O}(t)\right]_{K J}+\sum_{\nu} \dot{\mathbf{R}}_{\nu}(t) \cdot \mathbf{d}_{\nu ; K J}(t)\right) C_{J}^{s d}(t) .
\end{aligned}
$$

Each term in the above equation that depends on $\mathbf{R}(t)$ is to be evaluated along the trajectory, even though we removed the explicit dependence on $\mathbf{R}(t)$, while we kept the dependence on time.

Finally, we compute the time derivative of $\left|C_{K}^{s d}(t)\right|^{2}$ that will be used below when determining the expression of the classical force. The evolution of the electronic spin-diabatic 
population along a trajectory is

$$
\begin{aligned}
\frac{d\left|C_{K}^{s d}(t)\right|^{2}}{d t}= & \sum_{\nu} \frac{2 \mathcal{Q}_{\nu}(t)}{\hbar M_{\nu}} \cdot\left(\mathbf{f}_{\nu ; K}^{s d}(t)-\mathbf{A}_{\nu}(t)\right)\left|C_{K}^{s d}(t)\right|^{2} \\
& -2 \sum_{J} \sum_{\nu} \dot{\mathbf{R}}_{\nu}(t) \cdot \operatorname{Re}\left[\left[C_{K}^{s d}(t)\right]^{*} C_{J}^{s d}(t) \mathbf{d}_{\nu ; K J}(t)\right] \\
& +\frac{2}{\hbar} \sum_{J} \operatorname{Im}\left[\left[C_{K}^{s d}(t)\right]^{*} C_{J}^{s d}(t)\left[\hat{H}_{S O}(t)\right]_{K J}\right] .
\end{aligned}
$$

Summing this expression over $K$ yields zero, proving that the norm of the electronic wavefunction is conserved over time along each trajectory. The PNC imposed in Eq. (4) $\forall \mathbf{R}, t$ remains valid $\forall \mathbf{R}(t), t$ and even when the approximations (i), (ii) and (iii) above are introduced.

In Eqs. (35) we can then identify three contributions

$$
\frac{d\left|C_{K}^{s d}(t)\right|^{2}}{d t}=\left.\frac{d\left|C_{K}^{s d}(t)\right|^{2}}{d t}\right|_{\mathrm{QM}}+\left.\frac{d\left|C_{K}^{s d}(t)\right|^{2}}{d t}\right|_{\mathrm{NAC}}+\left.\frac{d\left|C_{K}^{s d}(t)\right|^{2}}{d t}\right|_{\mathrm{SO}}
$$

from the quantum momentum (QM), in Eq. (35a), from the NAC vectors (NAC), in Eq. (35b), and from the SOC matrix elements (SOC), in Eq. (35c).

\section{Electronic dynamics: Spin-adiabatic representation}

We use the expression of $\Phi_{\mathbf{R}(t)}(\mathbf{x}, t)$ in the spin-adiabatic basis, that follows from Eq. (13),

$$
\Phi_{\mathbf{R}(t)}(\mathbf{x}, t)=\sum_{j} C_{j}^{s a}(\mathbf{R}(t), t) \varphi_{\mathbf{R}(t)}^{(j)}(\mathbf{x})
$$


We insert this expression into the evolution equation (21) and following the same procedure described in the previous section we arrive at the final result

$$
\dot{C}_{k}^{s a}(t)=\left[-\frac{i}{\hbar} \epsilon_{s a}^{(k)}(t)+\sum_{\nu} \frac{\mathcal{Q}_{\nu}(t)}{\hbar M_{\nu}} \cdot\left(\mathbf{f}_{\nu ; k}^{s a}(t)-\mathbf{A}_{\nu}(t)\right)\right] C_{k}^{s a}(t)-\sum_{j} \sum_{\nu} \dot{\mathbf{R}}_{\nu}(t) \cdot \mathbf{d}_{\nu ; k j}(t) C_{j}^{s a}(t)
$$

where, as before, each term that depends on $\mathbf{R}(t)$ is to be evaluated along the trajectory even if it is not explicitly indicated here. This electronic equation in the spin-adiabatic basis is the equation that has been introduced in the original derivation of CT-MQC. .25

Similarly to Eqs. (35) we find that the population of a spin-adiabatic state evolves in time as

$$
\begin{aligned}
\frac{d\left|C_{k}^{s a}(t)\right|^{2}}{d t}= & \sum_{\nu} \frac{2 \mathcal{Q}_{\nu}(t)}{\hbar M_{\nu}} \cdot\left(\mathbf{f}_{\nu ; k}^{s a}(t)-\mathbf{A}_{\nu}(t)\right)\left|C_{k}^{s a}(t)\right|^{2} \\
& -2 \sum_{j} \sum_{\nu} \dot{\mathbf{R}}_{\nu}(t) \cdot \operatorname{Re}\left[\left[C_{k}^{s a}(t)\right]^{*} C_{j}^{s a}(t) \mathbf{d}_{\nu ; k j}(t)\right]
\end{aligned}
$$

which is basically identical to the expression in the spin-diabatic basis without the SOC. In fact, we identify here only two contributions of those pointed out in Eq. (36), namely

$$
\frac{d\left|C_{k}^{s a}(t)\right|^{2}}{d t}=\left.\frac{d\left|C_{k}^{s a}(t)\right|^{2}}{d t}\right|_{\mathrm{QM}}+\left.\frac{d\left|C_{k}^{s a}(t)\right|^{2}}{d t}\right|_{\mathrm{NAC}}
$$

In the spin-diabatic representation, Eqs. (34) above, and in the spin-adiabatic representation, Eq. (38), the evolution equations for the electronic coefficients are basically identical. The only difference is the presence of the SOC Hamiltonian in Eq. (34b), that disappears in Eq. (38) due to the diagonalization of the SOC contribution. In the general implementation of G-CT-MQC we will include the spin-orbit Hamiltonian matrix elements, but they will be identically zero in the spin-adiabatic basis because this contribution is absorbed in $\epsilon_{s a}^{(k)}(\mathbf{R}(t))$. This feature is highly desirable for implementation purposes, because the basic G-CT-MQC 
algorithm, i.e., the equations, is the same in both representations. We mention once more that the spin-adiabatic representation is a suitable choice when gradients of the SOC matrix elements are available from electronic-structure calculations, as for instance when using semiempirical methods. $\underline{63168}$

\section{Nuclear dynamics: Classical forces}

As mentioned above, fixing the gauge as indicated in Eq. (31) yields

$$
\dot{\mathbf{P}}_{\nu}(t)=\mathbf{F}_{\nu}(\mathbf{R}(t))=\frac{d \mathbf{A}_{\nu}(\mathbf{R}(t), t)}{d t}
$$

The expression of the time-dependent vector potential evaluated along the trajectory follows from its definition in Eq. (7), and using the spin-diabatic representation of the electronic wavefunction, we obtain

$$
\begin{aligned}
\mathbf{A}_{\nu}(\mathbf{R}(t), t)= & -i \hbar \sum_{J}\left[C_{J}^{s d}(\mathbf{R}(t), t)\right]^{*} \nabla_{\nu} C_{J}^{s d}(\mathbf{R}(t), t) \\
& -i \hbar \sum_{J, K}\left[C_{K}^{s d}(\mathbf{R}(t), t)\right]^{*} C_{J}^{s d}(\mathbf{R}(t), t) \mathbf{d}_{\nu ; K J}(\mathbf{R}(t)) .
\end{aligned}
$$

Since the time-dependent vector potential does not depend on the choice of the electronic representation, we did not use here the $s d$ label for $\mathbf{A}_{\nu}(\mathbf{R}(t), t)$. Furthermore, since the vector potential is a real quantity, we can rewrite Eqs. 42 as

$$
\begin{aligned}
\mathbf{A}_{\nu}(\mathbf{R}(t), t)= & \sum_{J}\left|C_{J}^{s d}(\mathbf{R}(t), t)\right|^{2} \nabla_{\nu} \gamma_{J}^{s d}(\mathbf{R}(t), t) \\
& +\hbar \sum_{J, K} \operatorname{Im}\left[\left[C_{K}^{s d}(\mathbf{R}(t), t)\right]^{*} C_{J}^{s d}(\mathbf{R}(t), t) \mathbf{d}_{\nu ; K J}(\mathbf{R}(t))\right]
\end{aligned}
$$

Henceforth, we will use a label $s d$ for the vector potential, making the distinction between the two representations, because in the different representations used to express it, the approximations have different effects, thus the final outcome is not the same. We will go 
back to this point below, when we will analyze numerical results.

Supposing that the NAC vectors are localized in space, we can observe that the first term in the expression of the vector potential dominates if compared to the second, since it contains an integral over time (we recall that $\nabla_{\nu} \gamma_{J}^{s d}(\mathbf{R}(t), t)=\mathbf{f}_{\nu ; J}^{s d}(\mathbf{R}(t))$ in Eq. (32c)), i.e., it accumulates over time. Therefore,

$$
\mathbf{A}_{\nu}^{s d}(\mathbf{R}(t), t) \simeq \sum_{J} \mathbf{f}_{\nu ; J}^{s d}(\mathbf{R}(t))\left|C_{J}^{s d}(\mathbf{R}(t), t)\right|^{2}
$$

and, using approximation (iii),

$$
\mathbf{F}_{\nu}^{s d}(t)=\sum_{J}\left(-\nabla_{\nu} \epsilon_{s d}^{(J)}(t)\right)\left|C_{J}^{s d}(t)\right|^{2}+\sum_{J} \mathbf{f}_{\nu ; J}^{s d}(t) \frac{d\left|C_{J}^{s d}(t)\right|^{2}}{d t}
$$

where we have dropped as done before the dependence on $\mathbf{R}(t)$ to make the expression more compact.

In the original derivation of CT-MQC, as described in Ref., 25 the expression of the force was given by directly evaluating the time derivative of the time-dependent vector potential, using Eq. (21), and by introducing, in a second step, the expansion of $\Phi_{\mathbf{R}(t)}$ in the adiabatic basis. An alternative procedure will be used here, analyzing the comparison between the spindiabatic and spin-adiabatic equations: such procedure allows us to determine the expression of the classical spin-diabatic force where gradients of the matrix elements of the spin-orbit Hamiltonian do not appear. This expression is highly suitable for future implementations in combination with quantum-chemistry codes that usually do not provide this information.

The expression of the spin-adiabatic force is

$$
\begin{aligned}
\mathbf{F}_{\nu}^{s a}(t)=\frac{d \mathbf{A}_{\nu}^{s a}(t)}{d t} & =\sum_{j}\left(-\nabla_{\nu} \epsilon_{s a}^{(j)}(t)\right)\left|C_{j}^{s a}(t)\right|^{2}+\sum_{j} \mathbf{f}_{\nu ; j}^{s a}(t) \frac{d\left|C_{j}^{s a}(t)\right|^{2}}{d t} \\
& =\mathbf{F}_{\nu, \mathrm{mf}}^{s a}(t)+\mathbf{F}_{\nu, \mathrm{NAC}}^{s a}(t)+\mathbf{F}_{\nu, \mathrm{qm}}^{s a}(t)
\end{aligned}
$$


where the mean-force (mf) term, the term containing the NAC vectors, and the quantummomentum (qm) term follow from Refs. $\frac{24}{26}$ and are

$$
\begin{aligned}
\mathbf{F}_{\nu, \mathrm{mf}}^{s a}(t) & =\sum_{j}\left|C_{j}^{s a}(t)\right|^{2}\left(-\nabla_{\nu} \epsilon_{s a}^{(j)}(t)\right) \\
\mathbf{F}_{\nu, \mathrm{NAC}}^{s a}(t) & =\sum_{j, k}\left[C_{j}^{s a}(t)\right]^{*} C_{k}^{s a}(t)\left(\epsilon_{s a}^{(j)}(t)-\epsilon_{s a}^{(k)}(t)\right) \mathbf{d}_{\nu ; j k}(t) \\
\mathbf{F}_{\nu, \mathrm{qm}}^{s a}(t) & =\sum_{j}\left|C_{j}^{s a}(t)\right|^{2}\left(\sum_{\nu^{\prime}} \frac{2 \mathcal{Q}_{\nu^{\prime}}(t)}{\hbar M_{\nu^{\prime}}} \cdot \mathbf{f}_{\nu^{\prime} ; j}^{s a}(t)\right)\left(\mathbf{f}_{\nu ; j}^{s a}(t)-\mathbf{A}_{\nu}^{s a}(t)\right)
\end{aligned}
$$

respectively. Note that in previous work, 2430 the sum of the first two contributions, i.e., mf and NAC terms, have been identified as the Ehrenfest force.

Using the similarity of Eq. (45) and Eq. (46a), and the similarity of Eq. (36) and Eq. (40), we can write the spin-diabatic force as

$$
\mathbf{F}_{\nu}^{s d}(t)=\mathbf{F}_{\nu, \mathrm{mf}}^{s d}(t)+\mathbf{F}_{\nu, \mathrm{NAC}}^{s d}(t)+\mathbf{F}_{\nu, \mathrm{qm}}^{s d}(t)+\mathbf{F}_{\nu, \mathrm{SO}}^{s d}(t)
$$

where last term is

$$
\begin{aligned}
\mathbf{F}_{\nu, \mathrm{SO}}^{s d}(t) & =\left.\sum_{K} \mathbf{f}_{\nu ; K}^{s d}(t) \frac{d\left|C_{K}^{s d}(t)\right|^{2}}{d t}\right|_{\mathrm{SO}} \\
& =\frac{2}{\hbar} \sum_{K, J} \operatorname{Im}\left[\left[C_{K}^{s d}(t)\right]^{*} C_{J}^{s d}(t)\left[\hat{H}_{S O}(t)\right]_{K J}\right] \mathbf{f}_{\nu ; K}^{s d}(t)
\end{aligned}
$$

\section{G-CT-MQC equations}

The G-CT-MQC equations that have been implemented for the numerical studies proposed in this work are summarized in this section. We will use the index $\alpha$ to explicitly indicate the dependence on the trajectory, that replaces the dependence on $\mathbf{R}(t)$ used above. Furthermore, since we will now provide equations that are valid in both electronic representations, we will drop the superscripts $s d$ and $s a$. In the above sections we used the indices $J, K$ to label the spin-diabatic states (we recall that $J$ stands for $S_{J}, M_{S_{J}}$, and similarly $K$ ), and the 
indices $j, k$ to label the spin-adiabatic states. Here, we will introduce the general symbols $m, n$ indicating the states in either basis, since we present equations that, formally, do not depend on the used electronic representation.

Electronic evolution is represented via an ordinary differential equation in time for the electronic coefficients, namely

$$
\begin{aligned}
\dot{C}_{m}^{\alpha}(t)= & {\left[-\frac{i}{\hbar} \epsilon^{(m), \alpha}(t)+\sum_{\nu} \frac{\mathcal{Q}_{\nu}^{\alpha}(t)}{\hbar M_{\nu}} \cdot\left(\mathbf{f}_{\nu ; m}^{\alpha}(t)-\mathbf{A}_{\nu}^{\alpha}(t)\right)\right] C_{m}^{\alpha}(t) } \\
& -\sum_{n}\left(\frac{i}{\hbar}\left[\hat{H}_{S O}^{\alpha}(t)\right]_{m n}+\sum_{\nu} \dot{\mathbf{R}}_{\nu}^{\alpha}(t) \cdot \mathbf{d}_{\nu ; m n}^{\alpha}(t)\right) C_{n}^{\alpha}(t),
\end{aligned}
$$

that is solved along a trajectory $\alpha$, generated by the force

$$
\mathbf{F}_{\nu}^{\alpha}(t)=\mathbf{F}_{\nu, \mathrm{mf}}^{\alpha}(t)+\mathbf{F}_{\nu, \mathrm{NAC}}^{\alpha}(t)+\mathbf{F}_{\nu, \mathrm{qm}}^{\alpha}(t)+\mathbf{F}_{\nu, \mathrm{SO}}^{\alpha}(t)
$$

whose terms are

$$
\begin{aligned}
\mathbf{F}_{\nu, \mathrm{mf}}^{\alpha}(t) & =\sum_{m}\left|C_{m}^{\alpha}(t)\right|^{2}\left(-\nabla_{\nu} \epsilon^{(m), \alpha}(t)\right) \\
\mathbf{F}_{\nu, \mathrm{NAC}}^{\alpha}(t) & =\sum_{m, n} \operatorname{Re}\left[\left[C_{m}^{\alpha}(t)\right]^{*} C_{n}^{\alpha}(t)\right]\left(\epsilon^{(m), \alpha}(t)-\epsilon^{(n), \alpha}(t)\right) \mathbf{d}_{\nu ; m n}(t) \\
\mathbf{F}_{\nu, \mathrm{qm}}^{\alpha}(t) & =\sum_{m}\left|C_{m}^{\alpha}(t)\right|^{2}\left(\sum_{\nu^{\prime}} \frac{2 \mathcal{Q}_{\nu^{\prime}}^{\alpha}(t)}{\hbar M_{\nu}} \cdot \mathbf{f}_{\nu^{\prime} ; m}^{\alpha}(t)\right)\left(\mathbf{f}_{\nu ; m}^{\alpha}(t)-\mathbf{A}_{\nu}^{\alpha}(t)\right) \\
\mathbf{F}_{\nu, \mathrm{SO}}^{\alpha}(t) & =\frac{2}{\hbar} \sum_{m} \sum_{n} \operatorname{Im}\left[\left[C_{m}^{\alpha}(t)\right]^{*} C_{n}^{\alpha}(t)\left[\hat{H}_{S O}(t)\right]_{m n}\right] \mathbf{f}_{\nu ; m}^{\alpha}(t) .
\end{aligned}
$$

In Eq. $55 \mathrm{~b}$ we assume that the NAC vectors are real. We recall that the velocity of trajectory $\alpha$ is

$$
\dot{\mathbf{R}}_{\nu}^{\alpha}(t)=\frac{\mathbf{P}_{\nu}^{\alpha}(t)}{M_{\nu}}
$$




\section{Numerical results}

In this section we employ the model systems proposed in Ref. $\frac{39}{30}$ to analyze exact quantum dynamics and discuss the approximations introduced in the previous sections for the derivation of G-CT-MQC equations in the spin-diabatic and spin-adiabatic representations. Afterwards, we compare quantum and trajectory-based electronic and nuclear dynamics in the two representations to test the performance of the G-CT-MQC algorithm.

\section{One-singlet/one-triplet model: Analysis of exact dynamics}

The first system, used as well in our previous work,,$\underline{30}$ is a one-dimensional (in nuclear space) one-singlet/one-triplet model, representing a singlet state $\left|\varphi_{R}^{(S, 0)}\right\rangle$ interacting with a triplet, whose components are $\left|\varphi_{R}^{(T, \pm 1)}\right\rangle$ and $\left|\varphi_{R}^{(T, 0)}\right\rangle$. The states are labeled according to the value of the projection of the spin along the $z$-axis. The Hamiltonian in the spin-diabatic basis is

$$
\hat{H}_{e l}(R)+\hat{H}_{S O}(R)=\left(\begin{array}{cccc}
\epsilon_{s d}^{(S)}(R) & z(R) & i b(R) & z^{*}(R) \\
z^{*}(R) & \epsilon_{s d}^{(T)}(R) & 0 & 0 \\
-i b(R) & 0 & \epsilon_{s d}^{(T)}(R) & 0 \\
z(R) & 0 & 0 & \epsilon_{s d}^{(T)}(R)
\end{array}\right)
$$

The diagonal elements in Eq. (57) are the energies of the singlet, $\epsilon_{s d}^{(S)}(R)$, and of the triplet, $\epsilon_{s d}^{(T)}(R)$, while the off-diagonal elements are the SOC between the singlet and the triplet, and there is no interaction within the multiplet. The elements of the matrix (57) are

$$
\begin{aligned}
\epsilon_{s d}^{(S)}(R) & =a_{1} \exp \left[-\alpha_{1} R\right]+\Delta E \\
\epsilon_{s d}^{(T)}(R) & =a_{2} \exp \left[-\alpha_{2} R\right] \\
z(R) & =c_{1} \sigma(R) \\
b(R) & =c_{0} \sigma(R)
\end{aligned}
$$


with $a_{1}=0.03452 \mathrm{E}_{h}, a_{2}=0.5 \mathrm{E}_{h}, \Delta E=0.04 \mathrm{E}_{h}, \alpha_{1}=0.35 \mathrm{bohr}^{-1}, \alpha_{2}=0.25 \mathrm{bohr}^{-1}$, $c_{1}=(0.0005+i 0.0005) \mathrm{E}_{h}, c_{0}=0.001 \mathrm{E}_{h}$. The function $\sigma(R)$ is a sigmoid function

$$
\sigma(R)= \begin{cases}1 & \text { if } R \leq R_{\sigma}-\Delta R_{\sigma} / 2 \\ 4\left(\frac{R-R_{\sigma}}{\Delta R_{\sigma}}\right)^{3}-3 \frac{R-R_{\sigma}}{\Delta R_{\sigma}} & \text { if } R_{\sigma}-\Delta R_{\sigma} / 2<R<R_{\sigma}+\Delta R_{\sigma} / 2 \\ -1 & \text { if } R \geq R_{\sigma}+\Delta R_{\sigma} / 2,\end{cases}
$$

that changes sign at $R=R_{\sigma}$ (over a range defined by the parameter $\Delta R_{\sigma}=2.0$ bohr). In what follows, we will present results for $R_{\sigma}=8.0$ bohr and $R_{\sigma}=9.5 \mathrm{bohr}$, tuning the strength of the SOC to investigate the performance of the new trajectory-based algorithm in different regimes. In particular, for $R_{\sigma}=8.0$ bohr we will observe a larger population transfer between the spin-diabatic states than for $R_{\sigma}=9.5 \mathrm{bohr}$, when the nuclear wavepacket, or analogously the swarm of trajectories, reaches the crossing point between the spin-diabatic potential energy curves, located at 10.0 bohr. Conversely, for $R_{\sigma}=8.0 \mathrm{bohr}$ a weaker non-adiabatic coupling than for $R_{\sigma}=9.5$ bohr is produced (this can be verified in Fig. 1 where the spin-adiabatic potential curves for $R_{\sigma}=9.5$ bohr are closer in energy than those corresponding to $R_{\sigma}=8.0$ bohr in the region of crossing of the spin-diabatic curves).

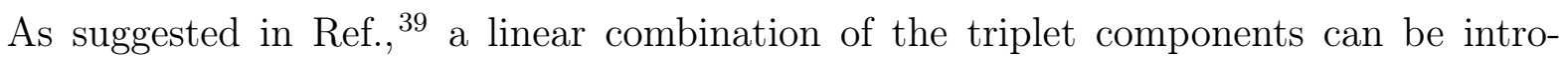
duced, and the new Hamiltonian can be block-diagonalized, which yields a new two-state Hamiltonian

$$
\hat{H}_{e l}(R)+\hat{H}_{S O}(R)=\left(\begin{array}{cc}
\epsilon_{s d}^{(S)}(R) & \sqrt{2\left|c_{1}\right|^{2}+c_{0}^{2}} \sigma(R) \\
\sqrt{2\left|c_{1}\right|^{2}+c_{0}^{2}} \sigma(R) & \epsilon_{s d}^{(T)}(R)
\end{array}\right)
$$

in the basis formed by the singlet $\left|\varphi_{R}^{(S, 0)}\right\rangle$ and the "triplet" $\left|\varphi_{R}^{\left(T_{S}\right)}\right\rangle$ states, with

$$
\left|\varphi_{R}^{\left(T_{S}\right)}\right\rangle=\frac{1}{\sqrt{2\left|c_{1}\right|^{2}+c_{0}^{2}}}\left(c_{1}\left|\varphi_{R}^{(T,-1)}\right\rangle+i c_{0}\left|\varphi_{R}^{(T, 0)}\right\rangle+c_{1}^{*}\left|\varphi_{R}^{(T, 1)}\right\rangle\right)
$$

Diagonal, $\epsilon_{s d}^{(S)}(R)$ and $\epsilon_{s d}^{(T)}(R)$, and off-diagonal, SOC, elements of matrix 60 are shown in 
Fig. 1 for $R_{\sigma}=8.0 \mathrm{bohr}$ (left panel) and $R_{\sigma}=9.5 \mathrm{bohr}$ (right panel). Diagonalization of the Hamiltonian yields the spin-adiabatic potential energy curves, $\epsilon_{s a}^{(1)}(R)$ and $\epsilon_{s a}^{(2)}(R)$, also shown in Fig. 1 .
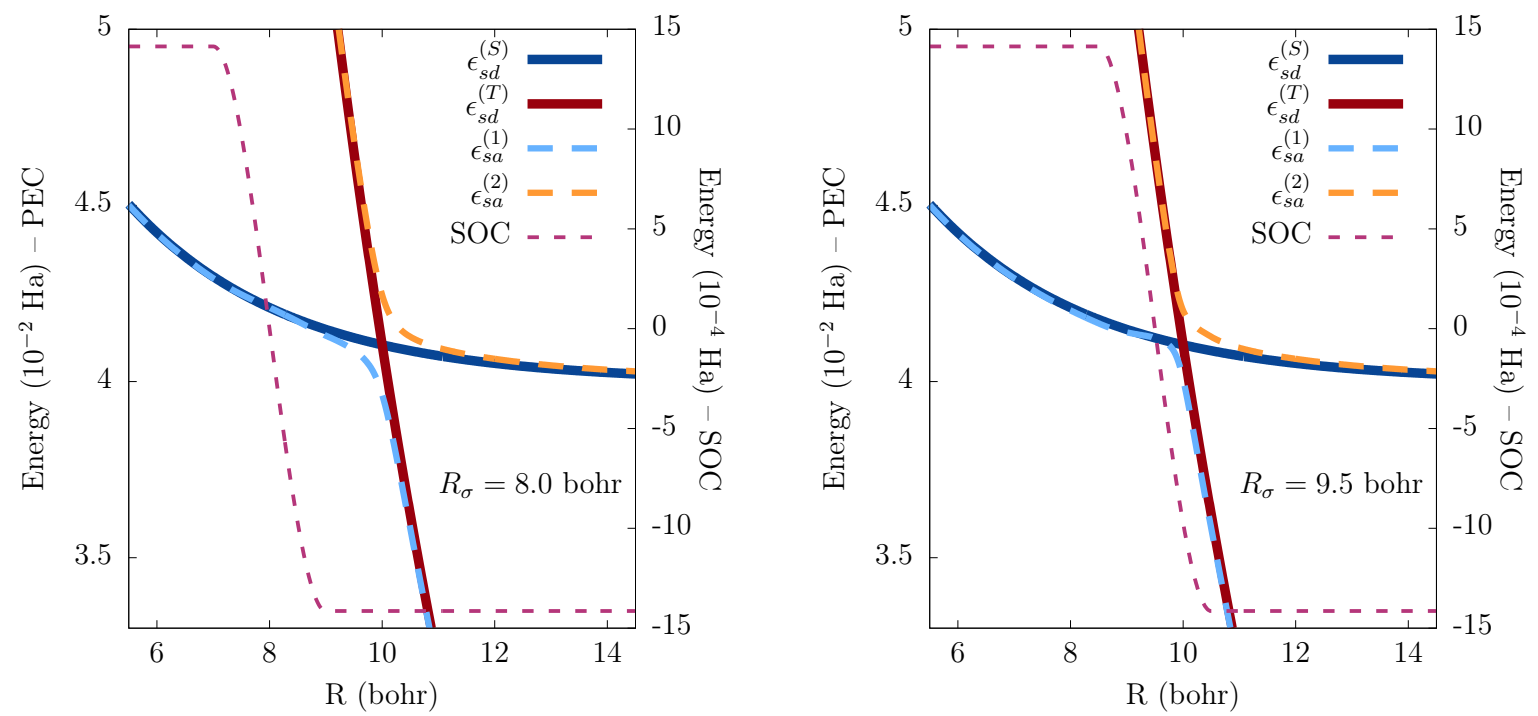

Figure 1: Spin-diabatic (blue lines, $\epsilon_{s d}^{(S)}$, and dark-red lines, $\epsilon_{s d}^{(T)}$ ) and spin-adiabatic (lightblue dashed lines, $\epsilon_{s a}^{(1)}$, and orange dashed lines, $\left.\epsilon_{s a}^{(2)}\right)$ potential energy curves (PECs) for $R_{\sigma}=8.0 \mathrm{bohr}$ (left panel) and $R_{\sigma}=9.5 \mathrm{bohr}$ (right panel) for the two-state model described by the Hamiltonian $(60)$. Rose dashed lines are the spin-orbit coupling (SOC), i.e., the offdiagonal elements of the Hamiltonian $(60)$.

For both values of $R_{\sigma}$, the dynamics is initiated in the lowest state at $R<R_{\sigma}$, i.e., the singlet $\left|\varphi_{R}^{(S)}\right\rangle$ in the spin-diabatic representation or the ground state $\left|\varphi_{R}^{(1)}\right\rangle$ in the spinadiabatic representation,

$$
|\Psi(R, t=0)\rangle=\chi(R, t=0)\left|\varphi_{R}^{(S)}\right\rangle=\chi(R, t=0)\left|\varphi_{R}^{(1)}\right\rangle
$$

At the initial time the nuclear wavefunction is the same in the two representations, and it is chosen to be a normalized Gaussian centered in $R_{0}=5.0$ bohr with variance $\sigma=\sqrt{0.05}$ bohr

$$
\chi(R, t=0)=\sqrt[4]{\frac{1}{\pi \sigma^{2}}} \exp \left[-\frac{\left(R-R_{0}\right)^{2}}{2 \sigma^{2}}+\frac{i}{\hbar} P_{0} R\right]
$$

The mean initial momentum is $P_{0}=20 \mathrm{bohr}^{-1}$, and the nuclear mass is $M=20000$ (in 
electronic-mass units). The time-dependent Schrödinger equation is solved in the spindiabatic basis using the Chebychev scheme ${ }^{69170}$ with Hamiltonian 60 . More precisely, this scheme, implemented in the ElVibRot code, ${ }^{71}$ was used with time step $d t=10.0$ fs (about $413.4 \mathrm{au}$ ) with 2048 grid points associated to 2048 particle-in-a-box basis functions ${ }^{72}$ with $R \in[3 ., 40$.$] bohr. In G-CT-MQC calculations, a time step of d t=0.012$ fs is used with 500 trajectories distributed in position and momentum according to the Wigner distribution associated to Eq. (63). The velocity-Verlet algorithm is used to integrate nuclear equations in G-CT-MQC, whereas the fourth-order Runge-Kutta method is used for the electronic evolution $\frac{73}{7}$ given in Eqs. 53 .

For the reduced two-state one-singlet/one-triplet model with $R_{\sigma}=8.0 \mathrm{bohr}$, we analyze exact dynamics adopting the perspective of the exact factorization, namely we study the TDPES and the electronic coefficients of the spin-diabatic (Eq. 12) and spin-adiabatic (Eq. (13) ) expansions of the electronic wavefunction. We set the gauge - in exact calculations - such that the time-dependent vector potential is zero everywhere at all times. In addition to the results presented below, we provide Supporting Information that show the evolution of the exact TDPES compared to its spin-diabatic and spin-adiabatic approximations based on G-CT-MQC.

Since we have introduced Dirac's notation to represent the electronic states in this twostate model, and we deal with a one-dimensional problem in nuclear space, we rewrite here the electronic time-dependent state as

$$
\left|\Phi_{R}(t)\right\rangle=\sum_{J=S, T} C_{J}^{s d}(R, t)\left|\varphi_{R}^{(J)}\right\rangle=\sum_{j=1,2} C_{j}^{s a}(R, t)\left|\varphi_{R}^{(j)}\right\rangle
$$

We analyze the following term of the TDPES

$$
\epsilon_{G I}(R, t)=\left\langle\Phi_{R}(t)\left|\hat{H}_{e l}(R)\right| \Phi_{R}(t)\right\rangle+\left\langle\Phi_{R}(t)\left|\hat{H}_{S O}(R)\right| \Phi_{R}(t)\right\rangle=\epsilon_{G I}^{(1)}(R, t)+\epsilon_{G I}^{(2)}(R, t),
$$


where we use the label GI to indicate that they are gauge-invariant. Furthermore, we recall that $\epsilon_{G I}(R, t)$ does not depend on the chosen representation for the electronic wavefunction, thus the sum of the two terms $\epsilon_{G I}^{(1)}(R, t)$ and $\epsilon_{G I}^{(2)}(R, t)$ is the same in the spin-diabatic and in the spin-adiabatic basis. In the spin-diabatic representation, the Hamiltonian given in Eq. 60 is the sum of $\hat{H}_{e l}(R)$ and $\hat{H}_{S O}(R)$, where $\hat{H}_{S O}(R)$ contributes only the off-diagonal elements. Therefore, $\epsilon_{G I}^{(2)}(R, t)$ is identically zero everywhere and at all times in the spinadiabatic representation because it is absorbed in the diagonal elements after diagonalization. This observation shows that a single term in Eq. (65) may vary in different representations, only the sum is invariant.

Using the spin-diabatic representation, the terms of the GI part of the TDPES are

$$
\begin{aligned}
\epsilon_{G I, s d}^{(1)}(R, t) & =\sum_{J=S, T}\left|C_{J}^{s d}(R, t)\right|^{2} \epsilon_{s d}^{(J)}(R) \\
\epsilon_{G I, s d}^{(2)}(R, t) & =\sum_{J=S, T} \sum_{K \neq J}\left|C_{J}^{s d}(R, t)\right|\left|C_{K}^{s d}(R, t)\right| \operatorname{Re}\left[e^{\frac{i}{\hbar}\left[\gamma_{K}^{s d}(R, t)-\gamma_{J}^{s d}(R, t)\right]}\left[\hat{H}_{S O}(R)\right]_{J K}\right],
\end{aligned}
$$

whereas in the spin-adiabatic representation they become

$$
\begin{aligned}
& \epsilon_{G I, s a}^{(1)}(R, t)=\sum_{j=1,2}\left|C_{j}^{s a}(R, t)\right|^{2} \epsilon_{s a}^{(j)}(R) \\
& \epsilon_{G I, s a}^{(2)}(R, t)=0 .
\end{aligned}
$$

In Eq. (66b) the term on the right-hand side contains an oscillatory contribution proportional to the off-diagonal elements of the Hamiltonian, the SOC. Since the full TDPES analyzed here does not present oscillations, as shown in Fig. 2 in the upper panels at three times along the dynamics, the term on the right-hand side of Eq. 66a has to develop oscillations as well. Clearly, the source of these oscillations is $\left|C_{J}^{s d}(R, t)\right|^{2}$ because $\epsilon_{s d}^{(J)}(R)$ is a smooth function of $R$, and this is shown as well in Fig. 2 (lower panels) at different times. To understand the nature of the oscillations in $\left|C_{J}^{s d}(R, t)\right|^{2}$ one can simply analyze the timedependent Schrödinger equation for the electron-nuclear state $|\Psi(R, t)\rangle$ in the spin-diabatic 


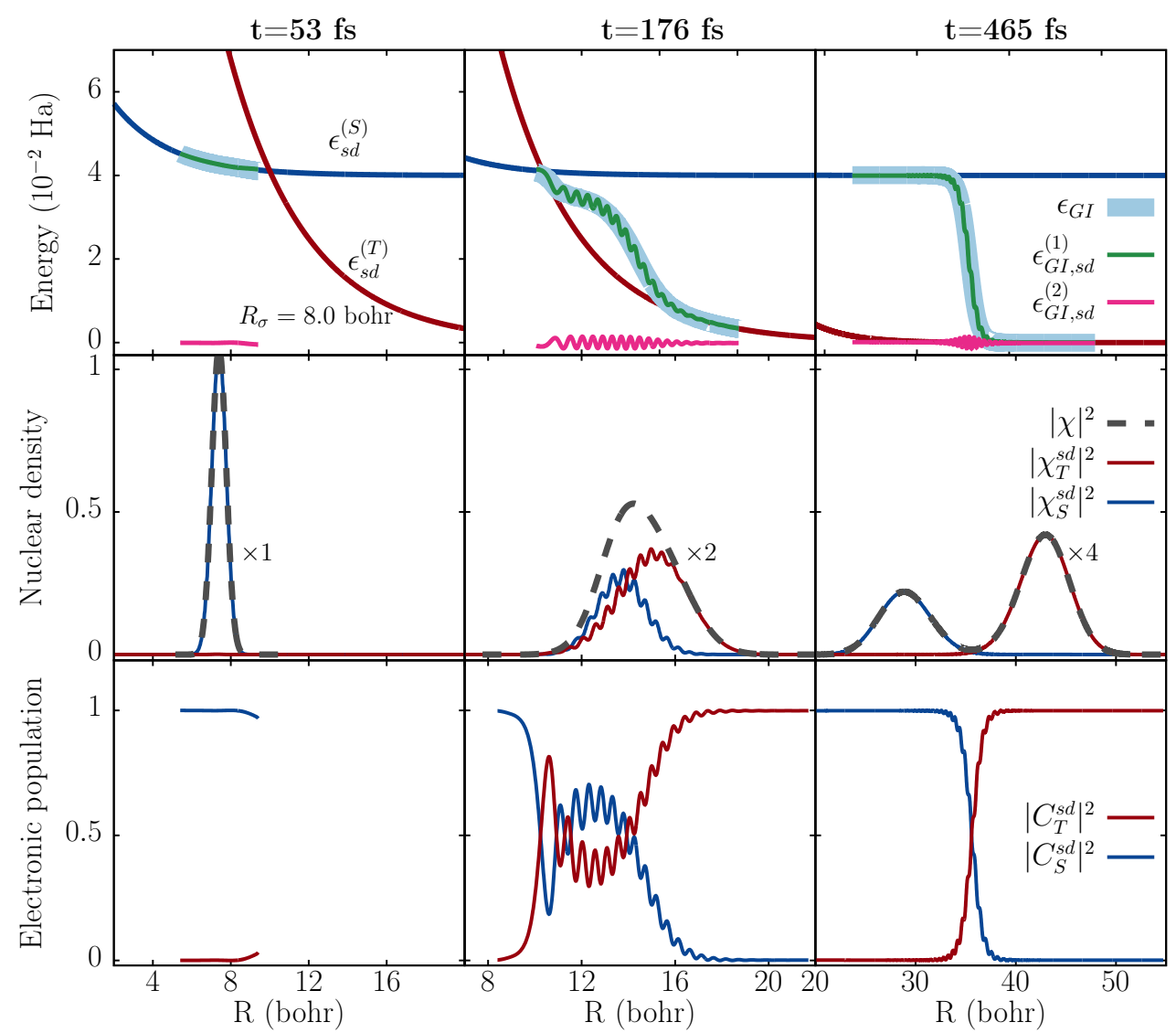

Figure 2: Upper panels: Spin-diabatic potential energy curves (blue lines, $\epsilon_{s d}^{(S)}$, and dark-red lines, $\left.\epsilon_{s d}^{(T)}\right)$; GI part of the TDPES given in Eq. 65) (light-blue thick lines), which is the sum of $\epsilon_{G I, s d}^{(1)}(R, t)$ of Eq. 66a) (green lines) and $\epsilon_{G I, s d}^{(2)}(R, t)$ of Eq. 66b) (magenta lines). Middle panels: nuclear density $|\chi(R, t)|^{2}$ (dark-grey dashed lines), decomposed into its spin-diabatic components $\left|\chi_{S}^{s d}(R, t)\right|^{2}$ (singlet, blue lines) and $\left|\chi_{T}^{s d}(R, t)\right|^{2}$ (triplet, dark-red lines). Lower panels: squared moduli of the spin-diabatic electronic coefficients $\left|C_{S}^{s d}(R, t)\right|^{2}$ (singlet, blue lines) and $\left|C_{T}^{s d}(R, t)\right|^{2}$ (triplet, dark-red lines). Snapshots are taken at $t=53,176,465$ fs.

representation and make the connection to the exact factorization. According to Eqs. (14) and (3) we have

$$
|\Psi(R, t)\rangle=\sum_{J=S, T} \chi_{J}^{s d}(R, t)\left|\varphi_{R}^{(J)}\right\rangle=\chi(R, t)\left|\Phi_{R}(t)\right\rangle .
$$


From Eq. 64 it follows that $\chi_{J}^{s d}(R, t)=\chi(R, t) C_{J}^{s d}(R, t)$. Evolution of $|\Psi(R, t)\rangle$ is given by

$$
i \hbar \partial_{t}\left(\begin{array}{c}
\chi_{S}^{s d}(R, t) \\
\chi_{T}^{s d}(R, t)
\end{array}\right)=\left[\frac{-\hbar^{2} \partial_{R}^{2}}{2 M}+\left(\begin{array}{cc}
\epsilon_{s d}^{(S)}(R) & H_{S O}(R) \\
H_{S O}(R) & \epsilon_{s d}^{(T)}(R)
\end{array}\right)\right]\left(\begin{array}{c}
\chi_{S}^{s d}(R, t) \\
\chi_{T}^{s d}(R, t)
\end{array}\right)
$$

which we have written in matrix form (NAC between singlet and triplet is zero). Selecting the equation for the singlet component (the same is true for the triplet component), and isolating the purely imaginary terms, we can derive the expression for the density of the singlet, $\left|\chi_{S}^{s d}(R, t)\right|^{2}$, (or equivalently for triplet $\left.\left|\chi_{T}^{s d}(R, t)\right|^{2}\right)$, that is

$$
\begin{aligned}
\partial_{t}\left|\chi_{S}^{s d}(R, t)\right|^{2}= & -\partial_{R}\left(\frac{\partial_{R} s_{S}^{s d}(R, t)}{M}\left|\chi_{S}^{s d}(R, t)\right|^{2}\right) \\
& +\frac{2}{\hbar} H_{S O}(R)\left|\chi_{S}^{s d}(R, t)\right|\left|\chi_{T}^{s d}(R, t)\right| \sin \left(\frac{s_{T}^{s d}(R, t)-s_{S}^{s d}(R, t)}{\hbar}\right)
\end{aligned}
$$

where $s_{S}^{s d}(R, t) / \hbar$ and $s_{T}^{s d}(R, t) / \hbar$ are the phases corresponding to $\chi_{S}^{s d}(R, t)$ and $\chi_{T}^{s d}(R, t)$. The oscillatory term depending on the difference of the phases between the singlet and triplet components is proportional to the SOC, i.e., the off-diagonal elements of the electronic Hamiltonian, and to the overlap of the singlet and triplet "wavepackets" (in the equation only the moduli appear). Therefore, the oscillations in $\left|\chi_{S / T}^{s d}(R, t)\right|^{2}$ develop in time as long the wavepackets are overlapping, and they disappear when the two portions of the nuclear density separate in space. We clearly observe this behavior in the middle panels of Fig. 2 , and since the nuclear density $|\chi(R, t)|^{2}=\left|\chi_{S}^{s d}(R, t)\right|^{2}+\left|\chi_{T}^{s d}(R, t)\right|^{2}$ is smooth, oscillations have to appear in $\left|C_{S / T}^{s d}(R, t)\right|^{2}$ in virtue of the relation $\left|\chi_{S / T}^{s d}(R, t)\right|^{2}=|\chi(R, t)|^{2}\left|C_{S / T}^{s d}(R, t)\right|^{2}$.

These observations relate to the approximation (ii) introduced in the previous section when deriving the G-CT-MQC equations in the spin-diabatic representation, that is the neglect of the spatial derivative of $\left|C_{J}^{s d}(R, t)\right|$. This approximation loses accuracy over time, as soon as $\left|C_{J}^{s d}(R, t)\right|$ develops oscillations that cannot be reproduced via G-CT-MQC by construction. In particular, the oscillations observed in the exact $\epsilon_{G I, s d}^{(1)}(R, t)$ and $\epsilon_{G I, s d}^{(2)}(R, t)$ 


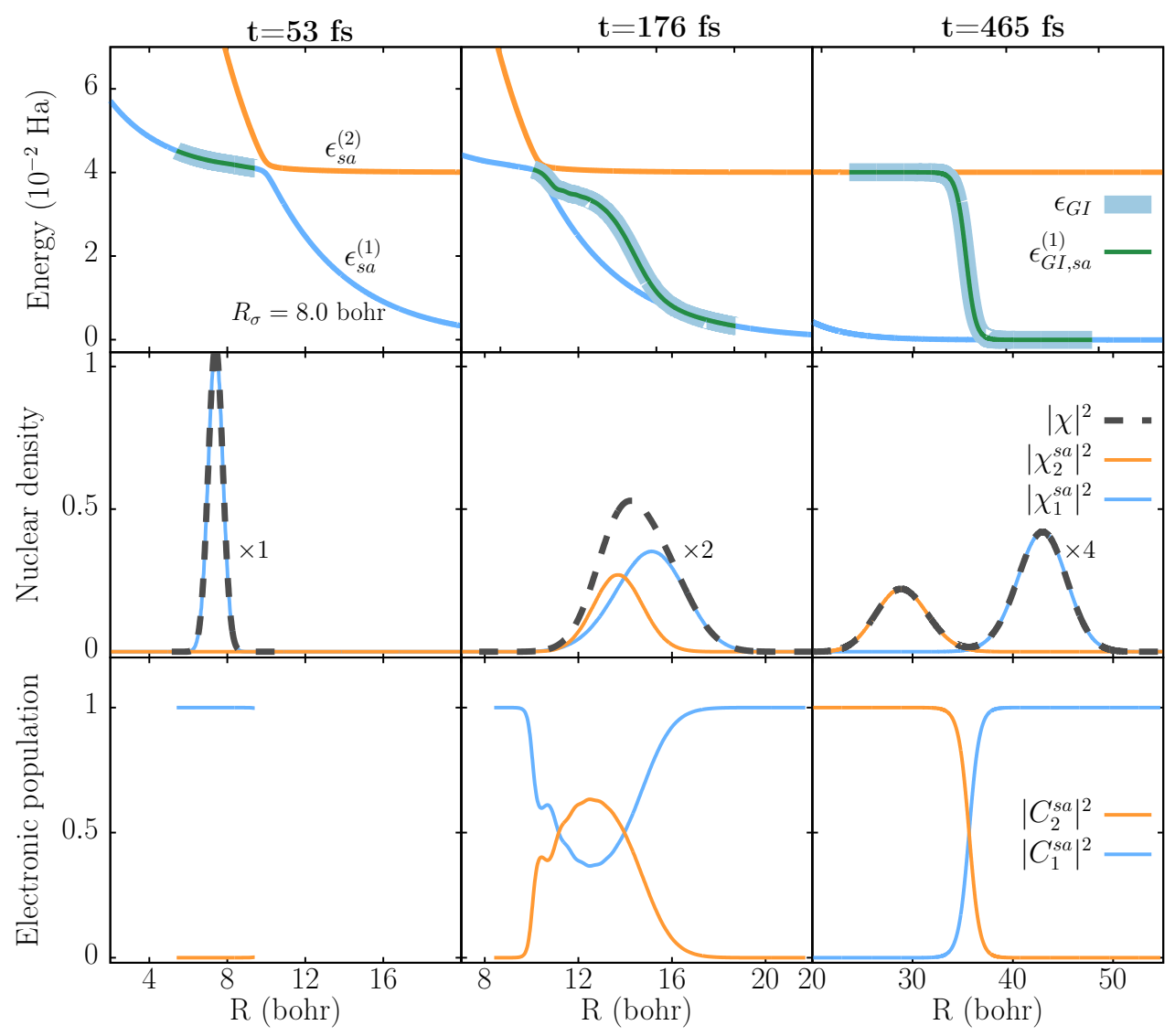

Figure 3: Upper panels: Spin-adiabatic potential energy curves (light-blue lines, $\epsilon_{s a}^{(1)}$, and orange lines, $\left.\epsilon_{s a}^{(2)}\right)$; GI part of the TDPES given in Eq. 65) (light-blue thick lines), which is identical to $\epsilon_{G I, s a}^{(1)}(R, t)$ of Eq. $67 \mathrm{a}$ (green lines). Middle panels: nuclear density $|\chi(R, t)|^{2}$ (dark-grey dashed lines), decomposed into its spin-adiabatic components $\left|\chi_{1}^{s a}(R, t)\right|^{2}$ (lightblue lines) and $\left|\chi_{2}^{s a}(R, t)\right|^{2}$ (orange lines). Lower panels: squared moduli of the spin-adiabatic electronic coefficients $\left|C_{1}^{s a}(R, t)\right|^{2}$ (light-blue lines) and $\left|C_{2}^{s a}(R, t)\right|^{2}$ (orange lines). Snapshots are taken at $t=53,176,465 \mathrm{fs}$. 
due to $\left|C_{J}^{s d}(R, t)\right|$ cannot be captured in the trajectory-based simulation, while the oscillatory behavior arising from the phases is still (approximately) reproduced. On the other hand, since in the spin-adiabatic representation $\left|C_{j}^{s a}(R, t)\right|$ has a slightly smoother shape as a function of $R$, and $\epsilon_{G I, s a}^{(1)}(R, t)$ does not develop oscillations (we remind that $\epsilon_{G I, s a}^{(2)}(R, t)=0$ in the spin-adiabatic representation), approximation (ii) is slightly more accurate. The shape of $\epsilon_{G I, s a}^{(1)}(R, t)$ is shown in Fig. 3 (upper panels) at three different times, together with the nuclear density and its decomposition in spin-adiabatic contributions (middle panels), and the squared moduli of the spin-adiabatic electronic coefficients (lower panels).

\section{One-singlet/one-triplet model: Nuclear dynamics and population analysis}

We compare here exact dynamics with G-CT-MQC results in the spin-diabatic and spinadiabatic basis for the one-singlet/one-triplet model reduced to a two-state problem.

Figure 4 shows the exact nuclear density compared with the histogram determined from the distribution of classical trajectories at three times along the dynamics (upper panels). At long times the G-CT-MQC spin-diabatic dynamics deviates from exact dynamics, since the approximations lose accuracy, and in fact we observe that the trajectories (pink histogram) move slightly faster than the quantum density (dashed lines) and than the trajectories propagated according to G-CT-MQC in the spin-adiabatic basis (green histogram). By contrast, a good agreement is observed at all times between exact results and the spin-adiabatic GCT-MQC results.

The trajectories evolve according to the force computed from the TDPES and the timedependent vector potential, which are computed at each time-step employing the electronic coefficients. Therefore, it is instructive to analyze the shape of those coefficients as functions of $R$ at the selected time snapshots. In particular, in Fig. 4 we show the squared modulus of the spin-diabatic coefficients $\left|C_{S / T}^{s d}(R, t)\right|^{2}$ (middle panels) and of the spin-adiabatic coefficients $\left|C_{1 / 2}^{s a}(R, t)\right|^{2}$ (lower panels) computed from exact propagation (lines) and from 


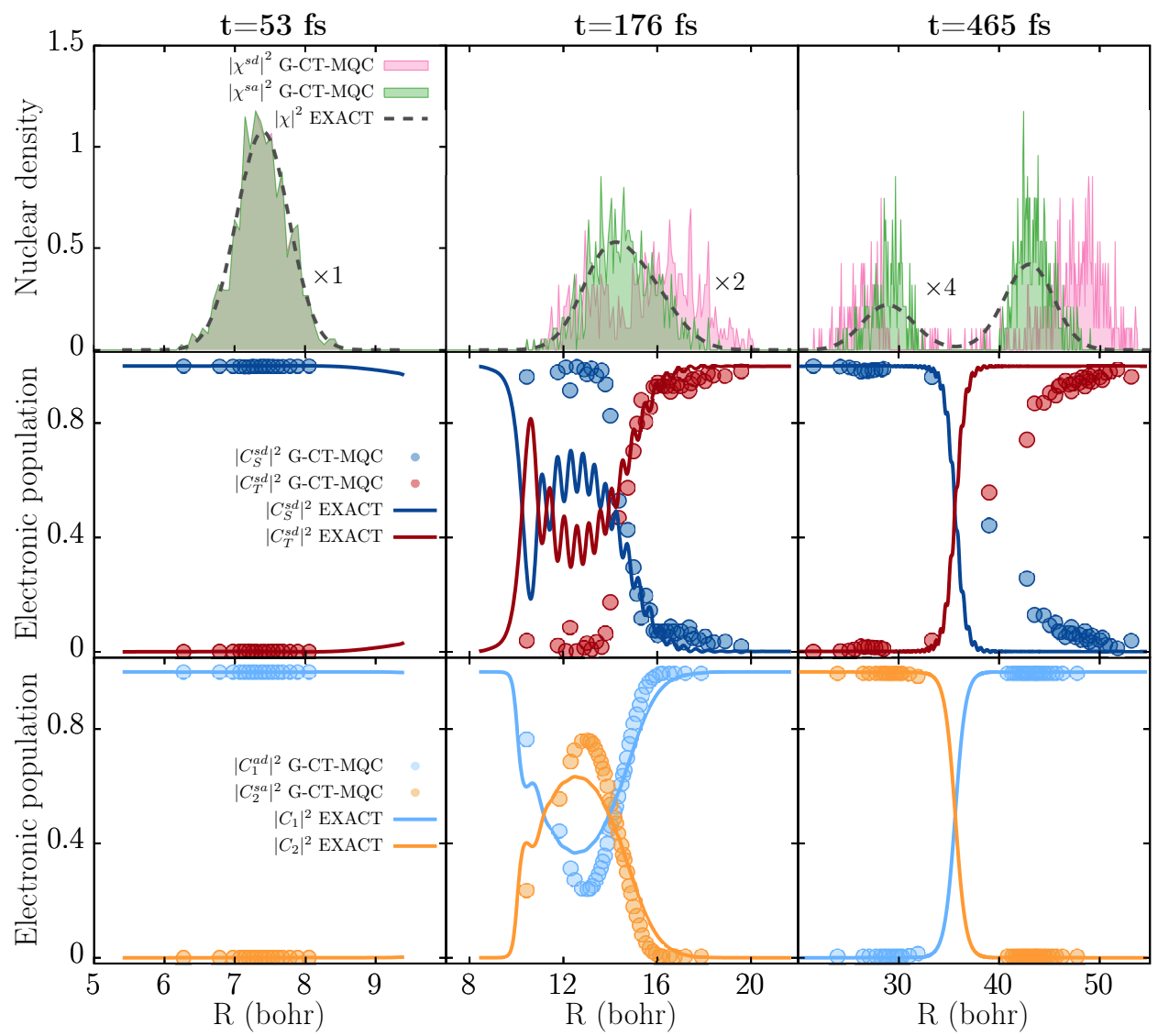

Figure 4: Two-state one-singlet/one-triplet model with $R_{\sigma}=8.0$ bohr. Upper panels: nuclear density from exact propagation (dark-grey dashed lines), and classical histograms constructed from the distributions of G-CT-MQC trajectories in the spin-diabatic (pink areas) and spin-adiabatic (green areas) representations. Middle panels: squared modulus of the spin-diabatic electronic coefficients $\left|C_{S / T}^{s d}(R, t)\right|^{2}$ (singlet, blue, and triplet, dark-red) from exact dynamics (lines) and G-CT-MQC (dots). Lower panels: squared modulus of the spinadiabatic electronic coefficients $\left|C_{1 / 2}^{s a}(R, t)\right|^{2}$ (ground state 1, light-blue, and excited state 2 , orange) from exact dynamics (lines) and G-CT-MQC (dots). Snapshots are taken at $t=53,176,465$ fs. 
G-CT-MQC (dots). Decoherence effects induced by the quantum-momentum term in the evolution equation (53a) yield the "sigmoid" shape of $\left|C_{S / T}^{s d}(R, t)\right|^{2}$ and $\left|C_{1 / 2}^{s a}(R, t)\right|^{2}$ at long times, because the electronic population along a single trajectory "collapses" to zero or to one, depending on which state the trajectory is associated to. However, it seems that decoherence effects appear too early in the spin-diabatic G-CT-MQC, and towards the end of the simulated dynamics they are not strong enough. From the point of view of the approximations introduced to derive G-CT-MQC, these issues are related to the fact that G-CT-MQC in the spin-diabatic representation does not capture properly the high-frequency oscillations observed in $\left|C_{S / T}^{s d}(R, t)\right|^{2}$ (see for instance the results at $176 \mathrm{fs}$ ) related to oscillations in the spin-diabatic nuclear densities, shown in Fig. 2 (middle panels). The spin-diabatic nuclear wavepackets are coupled along the dynamics via the potential coupling (the off-diagonal elements of the Hamiltonian), that is very different from the NAC in the spin-adiabatic representation. In general, in an adiabatic representation of the dynamics, we picture a nonadiabatic event as a nuclear wavepacket that passes through a region of large - localized - NAC, splits onto different states/potentials, and continues to evolve in a classical-like fashion on the new potentials (clearly, for the moment, in this picture we are discarding any recombination of wavepackets that might be the source of interferences). The corresponding behavior in a diabatic representation can be very different, and perhaps "more quantum" since the "interaction" between the different diabatic contributions of the wavepacket has an effect that is spatially-delocalized due to the shape of the potential coupling.

The spatially-delocalized nature of the potential - diabatic - coupling, that in our particular case is the SOC, is clearly an issue when adapting a trajectory-based algorithm designed

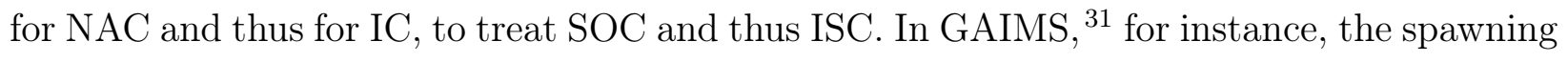
events take place in the regions where the SOC is large, but the SOC is "weighted" by the energy gap between the spin-diabatic energies - this is reminiscent of the expression of the NAC vectors where the energy gap appears at the denominator, and thus it becomes small where the potential energy surfaces are far apart. Note, however, that in GAIMS the SOC 
contribution to the quantum dynamics is always fully considered, that is, not only when the spin-diabatic potentials are close in energy. In generalized TSH (GTSH), proposed in Ref., 33 the fewest-switches procedure is applied in the spin-diabatic basis, but the SOC contribution to the hopping probability is considered only when the energy gap between the active state and the state towards which the hop should occur is smaller than a certain threshold. Another possibility is to "localize" the effect of the SOC-induced hops in TSH employing the Landau-Zener formula for determining the probability $(\mathrm{TSH} \otimes \mathrm{LZ}$ as dubbed by Tavernelli and co-workers in Ref. ${ }^{[32}$ ) in regions where the spin-diabatic potentials are close or cross. ${ }^{32}$ Therefore, the delocalized nature of the SOC is somehow bypassed in GTSH and TSH $\otimes \mathrm{LZ}$, and partially in GAIMS as well. In the spin-adiabatic representation, this issue does not appear because after diagonalization of the Hamiltonian all couplings become kinetic coupling, thus they are of NAC nature. In fact, for instance in the TSH-based approach proposed by Gonzalez and co-workers, $\stackrel{3438}{2}$ a diagonalization procedure is introduced such that the hopping probability is determined in the spin-adiabatic representation following the standard fewest-switches procedure, whereas nuclear dynamics is performed in the spin-diabatic representation, so as to circumvent calculations of gradients of the SOC in the classical force, which are usually not provided by quantum-chemistry codes. In the semi-empirical approach developed by Granucci and Persico ${ }^{63}$ for the electronic-structure part of the problem, instead, the whole TSH dynamics is consistently performed in the spin-adiabatic representation.

It is worth mentioning here an additional issue that appears in the framework of TSH: the sum of transition probabilities towards the components of a multiplet should be rotationally invariant, however, this requirement is not fulfilled by fewest-switches TSH in the spindiabatic representation. ${ }^{39} \mathrm{~A}$ solution to this problem is either considering an average SOC for each multiplet for the hopping ${ }^{[33}$ or treating the multiplets as a single state. ${ }^{35174}$

In G-CT-MQC the delocalized nature of the SOC is fully considered in Eqs. (53b) and (55d), however, these contributions to the evolution of the spin-diabatic electronic coefficients and to the force should naturally go to zero when decoherence effects appear. If the 
electronic coefficients correctly decay to zero/one, the SOC terms are zero even though the coupling itself remains non-zero. Our exact-factorization formulation of ICS and G-CT-MQC are shedding light on the physically-consistent procedure allowing us to achieve the expected results, by tackling the problem at the roots and without the need to introduce ad hoc strategies. The issue, nonetheless, remains, because if decoherence is not accurately reproduced in the spin-diabatic representation, spurious population transfers might be observed.
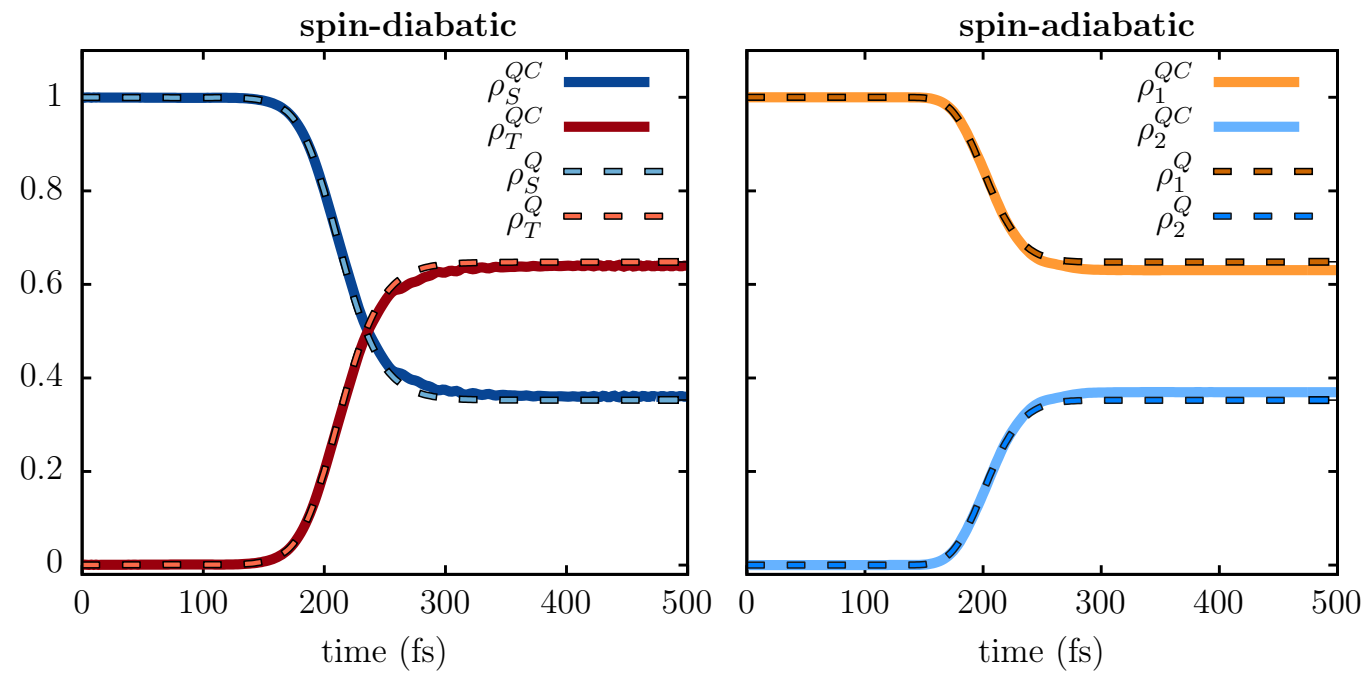

Figure 5: Two-state one-singlet/one-triplet model with $R_{\sigma}=8.0$ bohr. Left panel: Spindiabatic electronic populations from exact calculations (dashed lines) and G-CT-MQC (continuous lines). Right panel: Spin-adiabatic electronic populations from exact calculations (dashed lines) and G-CT-MQC (continuous lines).

In Fig. 5 we analyze this issue by showing the electronic populations, spin-diabatic and spin-adiabatic, determined by averaging $\left|C_{S / T}^{s d}(t)\right|^{2}$ and $\left|C_{1 / 2}^{s a}(t)\right|^{2}$ over the trajectories. Using the notation introduced in Eqs. (53) the quantum-classical $(Q C)$ population of state $m$ is estimated as

$$
\rho_{m}^{Q C}(t)=\frac{1}{N_{\text {traj }}} \sum_{\alpha=1}^{N_{\text {traj }}}\left|C_{m}^{\alpha}(t)\right|^{2}
$$

with $m=S, T$ or $m=1,2$. G-CT-MQC results are compared with the quantum $(Q)$ result

$$
\rho_{m}^{Q}(t)=\int d R\left|\chi_{m}(R, t)\right|^{2} .
$$


Despite the slight disagreement between exact and spin-diabatic G-CT-MQC calculations observed in Fig. 4, electronic populations are well reproduced all along the dynamics in both representations.

Similarly to Figs. 4 and 5, we compare G-CT-MQC results in the spin-diabatic representation and in the spin-adiabatic representation with exact calculations for the one-singlet/onetriplet model reduced to two electronic states with $R_{\sigma}=9.5$ bohr. Such value of $R_{\sigma}$ yields a weaker SOC between the two states. Fig. 6 shows the nuclear density (upper panels), the squared moduli of the spin-diabatic electronic coefficients (middle panels), and the squared moduli of the spin-adiabatic electronic coefficients (lower panels) at times $t=50,170,340 \mathrm{fs}$ along the dynamics. At long times, we observe that spin-diabatic propagation yields trajectories that move slightly faster than those obtained in the spin-adiabatic formulation of G-CT-MQC and than the exact density. This effect follows from the fact that G-CT-MQC does not capture exhaustively the high-frequency oscillations appearing in the squared moduli of the electronic coefficients, which is clear from the comparison of exact and G-CT-MQC results in the middle panel of Fig. 6 at time $t=140$ fs. However, the overall shape of the coefficients follows qualitatively the shape of the exact coefficients. As previously observed, in the spin-adiabatic representation, G-CT-MQC results are in better agreement with the exact ones. Analogously to the case $R_{\sigma}=8.0 \mathrm{bohr}$, the population of the electronic states, either in spin-diabatic or in the spin-adiabatic basis, are in very good agreement with exact calculations, as Fig. 7 shows, and we might actually claim that the populations of the spin-diabatic states are slightly better reproduced by G-CT-MQC than the populations of the spin-adiabatic states. 


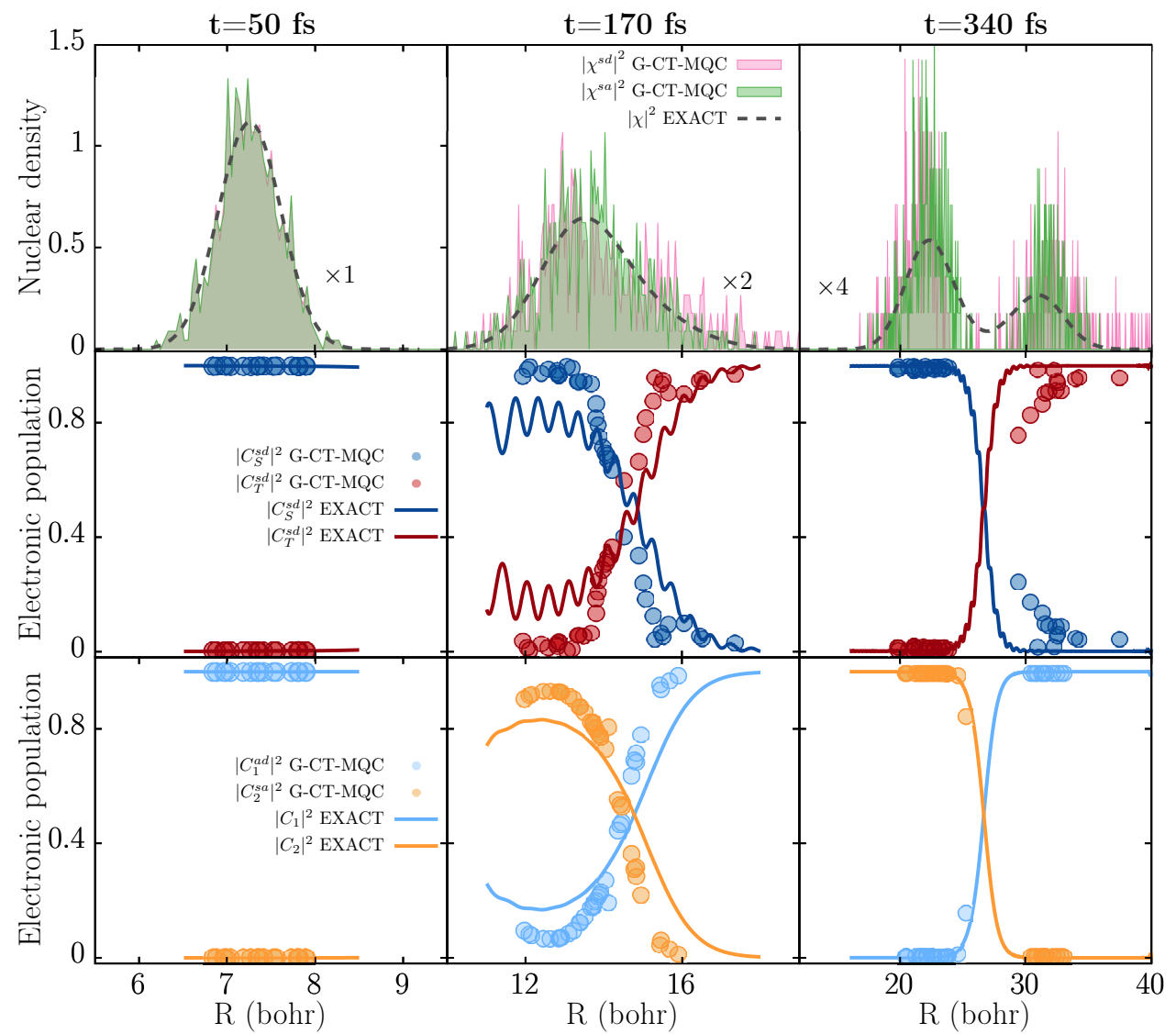

Figure 6: Two-state one-singlet/one-triplet model with $R_{\sigma}=9.5 \mathrm{bohr}$. The reported data are the same as in Fig. 4, but here snapshots are taken at $t=50,170,340 \mathrm{fs}$. 

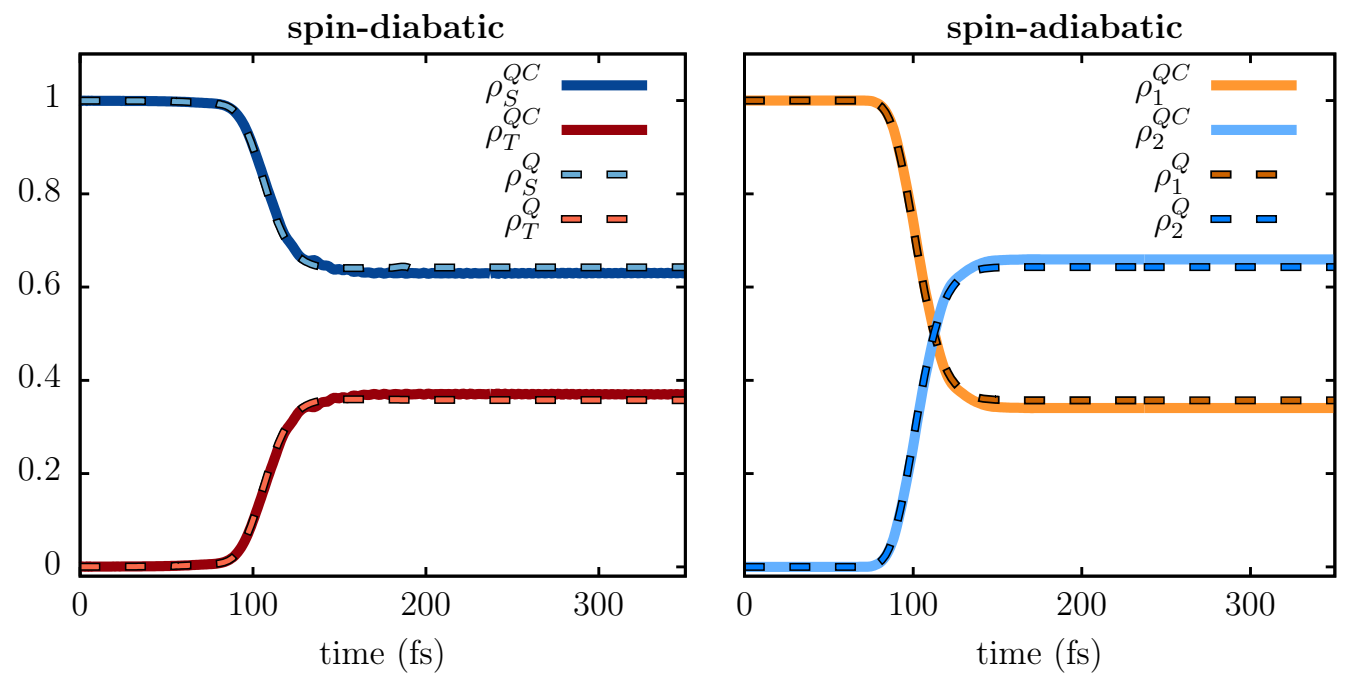

Figure 7: Two-state one-singlet/one-triplet model with $R_{\sigma}=9.5 \mathrm{bohr}$. Populations of the spin-diabatic (left panel) and spin-adiabatic (right panel) electronic states are shown as functions of time. The color code is the same as in Fig. 5.

\section{Two-singlet/one-triplet model: Nuclear dynamics and population analysis}

The second system under study has been proposed in Ref. .39 as well, and it involves two singlets and one triplet. The five-state Hamiltonian in the spin-diabatic basis reads

$$
\hat{H}_{e l}(R)+\hat{H}_{S O}(R)=\left(\begin{array}{ccccc}
\epsilon_{s d}^{\left(S_{1}\right)}(R) & z_{1} & i b_{1} & z_{1}^{*} & 0 \\
z_{1}^{*} & \epsilon_{s d}^{(T)}(R) & 0 & 0 & z_{2} \\
-i b_{1} & 0 & \epsilon_{s d}^{(T)}(R) & 0 & i b_{2} \\
z_{1} & 0 & 0 & \epsilon_{s d}^{(T)}(R) & z_{2}^{*} \\
0 & z_{2}^{*} & -i b_{2} & z_{2} & \epsilon_{s d}^{\left(S_{2}\right)}(R)
\end{array}\right) \text {, }
$$

where the states are indicated as $\left|\varphi_{R}^{\left(S_{1}, 0\right)}\right\rangle$ and $\left|\varphi_{R}^{\left(S_{2}, 0\right)}\right\rangle$ for the singlets, with spin-diabatic energies $\epsilon_{s d}^{\left(S_{1}\right)}(R)$ and $\epsilon_{s d}^{\left(S_{2}\right)}(R)$, respectively, and $\left|\varphi_{R}^{(T, \pm 1)}\right\rangle$ and $\left|\varphi_{R}^{(T, 0)}\right\rangle$ for the triplets, with spin-diabatic energy $\epsilon_{s d}^{(T)}(R)$. As for the previous model, we can define a linear combination of the triplets, i.e., $\left|\varphi_{R}^{\left(T_{S_{1}}\right)}\right\rangle$, that interacts with the singlet state $\left|\varphi_{R}^{\left(S_{1}, 0\right)}\right\rangle$ with coupling $\gamma_{1}$. An analogous procedure is used for the singlet $\left|\varphi_{R}^{\left(S_{2}, 0\right)}\right\rangle$ that interacts with $\left|\varphi_{R}^{\left(T_{S_{2}}\right)}\right\rangle$ with coupling 
$\gamma_{2}$. Note that in general $\left|\varphi_{R}^{\left(T_{S_{1}}\right)}\right\rangle \neq\left|\varphi_{R}^{\left(T_{S_{2}}\right)}\right\rangle$, and their overlap is

$$
\cos \phi_{12}=\left\langle\varphi_{R}^{\left(T_{S_{1}}\right)} \mid \varphi_{R}^{\left(T_{S_{2}}\right)}\right\rangle=\frac{2 \operatorname{Re}\left[z_{1} z_{2}^{*}\right]+b_{1} b_{2}}{\gamma_{1} \gamma_{2}}
$$

If $\phi_{12}=0, \pi$, the model reduces to a three-state problem because $\left|\varphi_{R}^{\left(T_{S_{1}}\right)}\right\rangle= \pm\left|\varphi_{R}^{\left(T_{S_{2}}\right)}\right\rangle$, whereas if $\phi_{12} \neq 0, \pi$ the model reduces to a four-state problem with orthogonal states $\left|\varphi_{R}^{\left(S_{1}, 0\right)}\right\rangle,\left|\varphi_{R}^{\left(T_{S_{1}}\right)}\right\rangle,\left|\tilde{\varphi}_{R}^{\left(T_{S_{2}}\right)}\right\rangle,\left|\varphi_{R}^{\left(S_{2}, 0\right)}\right\rangle$, with

$$
\left|\tilde{\varphi}_{R}^{\left(T_{S_{2}}\right)}\right\rangle=\frac{\left|\varphi_{R}^{\left(T_{S_{1}}\right)}\right\rangle-\left|\varphi_{R}^{\left(T_{S_{2}}\right)}\right\rangle \cos \phi_{12}}{\sin \phi_{12}} \quad \text { with } \phi_{12} \neq 0, \pi
$$

Using these four states as spin-diabatic basis, the Hamiltonian (73) reduces to

$$
\hat{H}_{e l}(R)+\hat{H}_{S O}(R)=\left(\begin{array}{cccc}
\epsilon_{s d}^{\left(S_{1}\right)}(R) & \gamma_{1} & 0 & 0 \\
\gamma_{1} & \epsilon_{s d}^{(T)}(R) & 0 & \gamma_{2} \cos \phi_{12} \\
0 & 0 & \epsilon_{s d}^{(T)}(R) & \gamma_{2} \sin \phi_{12} \\
0 & \gamma_{2} \cos \phi_{12} & \gamma_{2} \sin \phi_{12} & \epsilon_{s d}^{\left(S_{2}\right)}(R)
\end{array}\right)
$$

The effective coupling between the singlets $\left|\varphi_{R}^{\left(S_{1}, 0\right)}\right\rangle$ and $\left|\varphi_{R}^{\left(S_{2}, 0\right)}\right\rangle$ is mediated by the triplet and depends on the overlap $\cos \phi_{12}$. In the following we will use $\epsilon_{s d}^{\left(S_{1}\right)}(R)=\epsilon_{s d}^{(S)}(R)$ with $\epsilon_{s d}^{(S)}(R)$ given in Eq. 58a), and $\epsilon_{s d}^{\left(S_{2}\right)}(R)=\epsilon_{s d}^{\left(S_{1}\right)}(R)-\Delta E_{S}$ with $\Delta E_{S}=0.035 \mathrm{E}_{h}$; similarly $\epsilon_{s d}^{(T)}(R)$ is given in Eq. 58b. The couplings are chosen as $\gamma_{1}=\gamma_{2}=10^{-3} \sqrt{2} \mathrm{E}_{h}$ and we will consider the cases $\phi_{12}=0, \pi / 4, \pi / 2$. 1 Figure 8 shows the spin-diabatic potential energy curves, along with the spin-adiabatic energies obtained by diagonalization of the Hamiltonian (76), only for the case $\phi_{12}=\pi / 4$.

Initial conditions for the dynamics and computational details on the propagation in this reduced four-state model for the two-singlet/one-triplet problem are basically the same as for the one-singlet/one-triplet model. The only difference is that the initially populated

\footnotetext{
${ }^{1}$ In this model study, rather than tuning the strength of the spin-diabatic coupling, we analyze the effects of various couplings between the triplet components and the two singlets.
} 

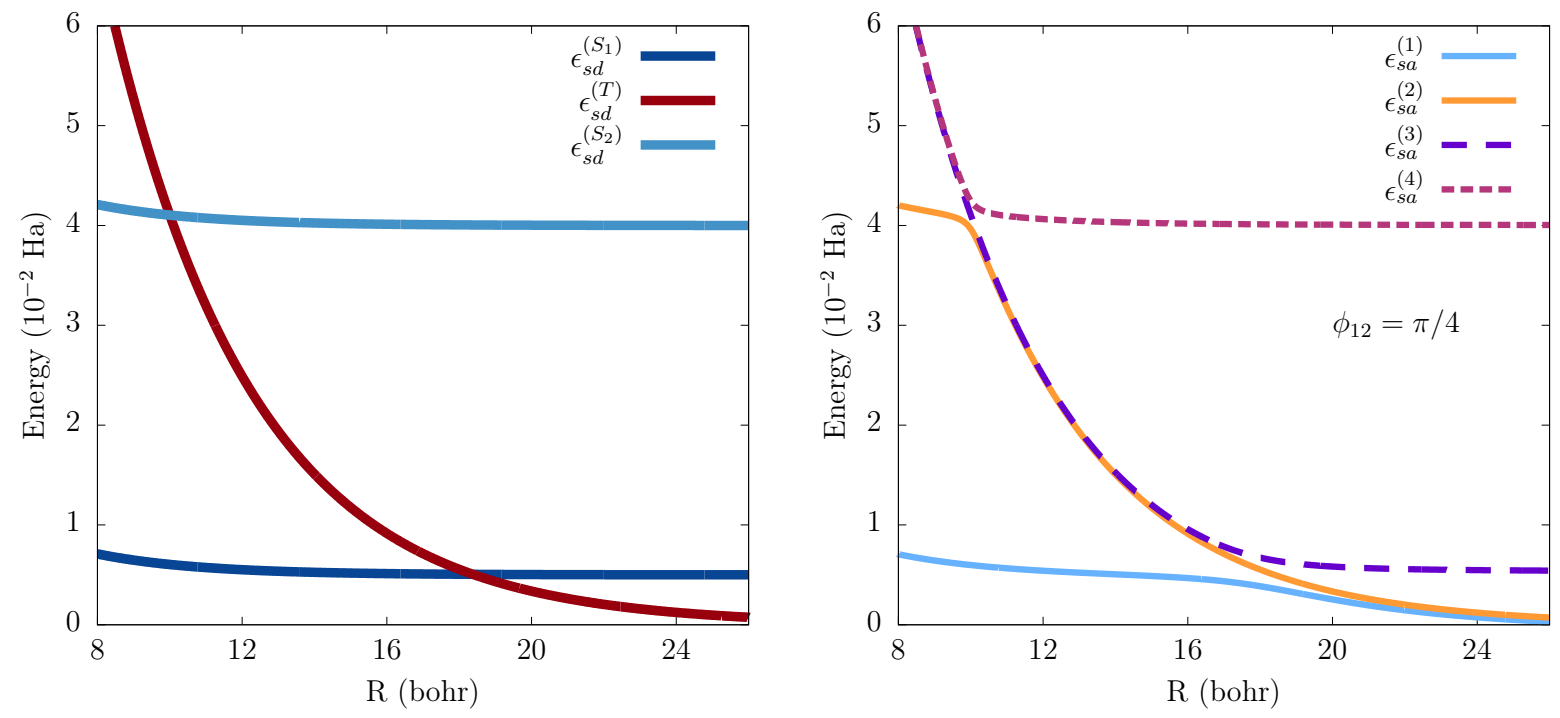

Figure 8: Left panel: Spin-diabatic (blue line, $\epsilon_{s d}^{\left(S_{1}\right)}$, dark-red line, $\epsilon_{s d}^{(T)}$, and light-blue line $\left.\epsilon_{s d}^{\left(S_{2}\right)}\right)$ potential energy curves for the four-state model described by the Hamiltonian (76). Right panel: Spin-adiabatic (light-blue continuous line, $\epsilon_{s a}^{(1)}$, orange continuous line, $\epsilon_{s a}^{(2)}$, purple dashed line, $\epsilon_{s a}^{(3)}$ and rose dashed line, $\left.\epsilon_{s a}^{(4)}\right)$ potential energy curves obtained by diagonalizing the Hamiltonian (76) with $\phi_{12}=\pi / 4$.

electronic state is the spin-diabatic $\left|\varphi_{R}^{\left(S_{2}\right)}\right\rangle$ or equivalently the spin-adiabatic $\left|\varphi_{R}^{(2)}\right\rangle$.

Similarly to what we presented for the one-singlet/one-triplet model, we compare in Fig. 9 nuclear dynamics from exact calculations and from G-CT-MQC in the spin-diabatic and spin-adiabatic basis. For the simulated dynamics, trajectories remain well localized in the region where the nuclear density is "large". However, in both representations and at the final time $(t=212 \mathrm{fs})$ we notice for all values of $\phi_{12}$ that the histograms slightly overestimate the splitting of the density, a feature that has been observed already in CT-MQC $48 \mid 64$ and that has been ascribed to the effect of the quantum-momentum. At the times reported in Fig. 9 the agreement between spin-diabatic and spin-adiabatic G-CT-MQC results seems better than in Figs. 4 and 6. However, we expect a similar deviation in this four-state model as in the two-state model at longer times, when the nuclear wavepackets, or equivalently the trajectoires, have reached the region where the potential energy curves are flat (meaning that the error might accumulate over time).

We report in Fig. 10 electronic populations as functions of time for different values of 


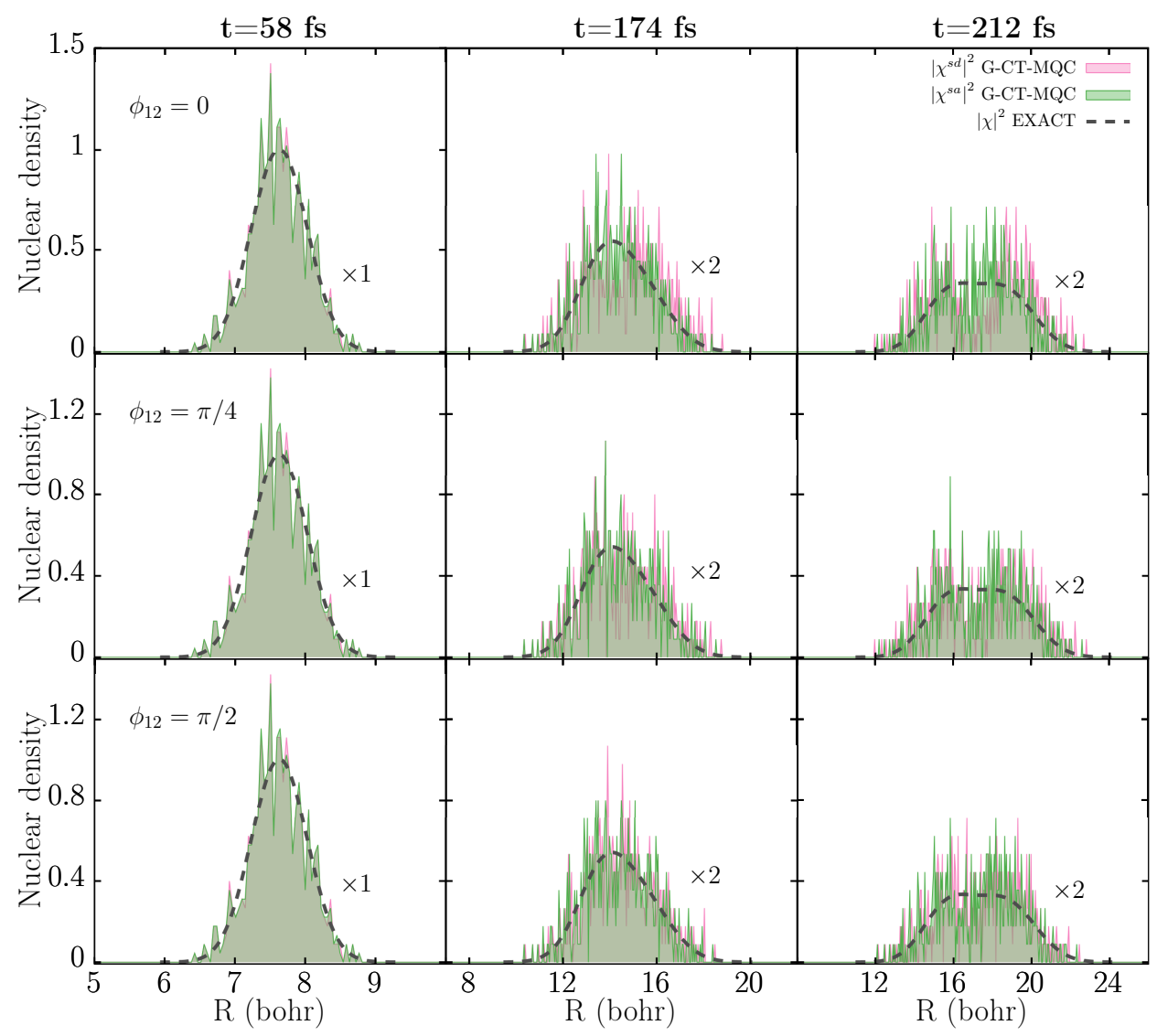

Figure 9: Nuclear density from exact propagation (dark-grey dashed lines), and classical histograms constructed from the distributions of G-CT-MQC trajectories in the spin-diabatic (pink areas) and spin-adiabatic (green areas) representations. Snapshots are taken at $t=$ $58,174,212$ fs for $\phi_{12}=0$ (upper panels), $\phi_{12}=\pi / 4$ (middle panels) and $\phi_{12}=\pi / 2$ (lower panels). 


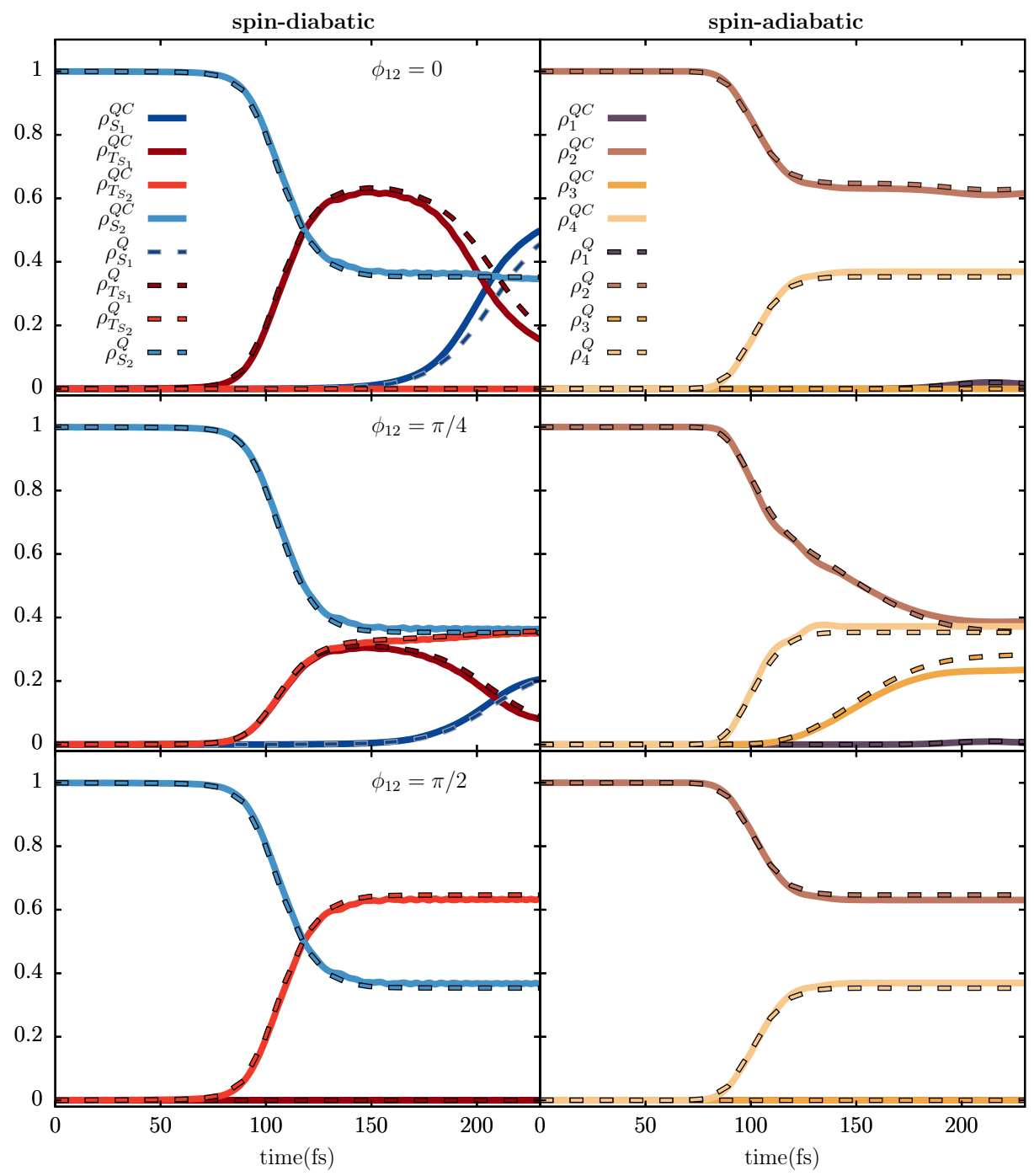

Figure 10: Two-singlet/one-triplet model reduced to a four-state problem. Left panels: Spin-diabatic electronic populations from exact calculations (dashed lines) and G-CT-MQC (continuous lines). Right panels: Spin-adiabatic electronic populations from exact calculations (dashed lines) and G-CT-MQC (continuous lines). Different values of the singlet/triplet coupling are used for $\phi_{12}=0$ (upper panels), $\phi_{12}=\pi / 4$ (central panels), and $\phi_{12}=\pi / 2$ (lower panels). 
the overlap between the triplet states, as indicated above. If $\phi_{12}=0$ (upper left panels in Fig. 10), as observed above, the model reduces to a three-state problem, where $\left|\varphi_{R}^{\left(T_{S_{1}}\right)}\right\rangle$ is populated when the trajectories (or the nuclear wavepacket) go through the first crossing at $R=11$ bohr. Later on, the triplet transfers population to $\left|\varphi_{R}^{\left(S_{1}\right)}\right\rangle$ at the crossing located at $R=18$ bohr between the triplet $\epsilon_{s d}^{(T)}(R)$ and singlet $\epsilon_{s d}^{\left(S_{1}\right)}(R)$ potential energy curves. If $\phi_{12}=$ $\pi / 4$ (middle left panels in Fig. 10), the four states are populated during the dynamics: at the

first crossing between $\epsilon_{s d}^{\left(S_{2}\right)}(R)$ and $\epsilon_{s d}^{(T)}(R)$, the triplets are populated; after the trajectories leave the crossing region, only the population of $\left|\varphi_{R}^{\left(T_{S_{1}}\right)}\right\rangle$ changes as effect of the transfer to $\left|\varphi_{R}^{\left(S_{1}\right)}\right\rangle$ at the second crossing between $\epsilon_{s d}^{(T)}(R)$ and $\epsilon_{s d}^{\left(S_{1}\right)}(R)$. If $\phi_{12}=\pi / 2$ (lower panels in Fig. 10, the model reduces to a two-state problem because only $\left|\varphi_{R}^{\left(S_{1}\right)}\right\rangle$ and $\left|\varphi_{R}^{\left(T_{S_{2}}\right)}\right\rangle$ are coupled and populated along the dynamics.

G-CT-MQC results shown in Fig. 10 reproduce well exact results in both representations (spin-diabatic, left panels, and spin-adiabatic, right panels), however, in the spin-diabatic representation agreement might be lost at longer times because spurious population transfer driven by the SOC is observed due to the lack of complete decoherence. This issue can be circumvented with ad hoc adjustments to the algorithm, for instance by switching off the effect of the SOC between two states in the electronic equation if the spin-diabatic energies corresponding to those states are far apart, as it is done routinely for other schemes. ${ }^{32133}$ This additional numerical developments are, however, beyond the scope of the work presented here, and will not be investigated further.

\section{Conclusions and perspectives}

We presented a detailed derivation of the generalized CT-MQC algorithm, dubbed G-CTMQC, to treat internal conversion and intersystem crossing processes within the approximate version of the exact factorization.

The approximations introduced to derive the algorithm in the spin-diabatic and spin- 
adiabatic representations are discussed and tested with numerical simulations of illustrative model systems. For one model, in particular, we analyzed exact quantum dynamics in the perspective of the exact factorization, in order to point out eventual sources of errors in the trajectory-based approach. In addition, we stressed the critical role of decoherence in regulating the long-range effect of spin-orbit coupling, that is usually very delocalized in space. In this context, we have discussed our approach in comparison to other trajectorybased methods designed to treat intersystem crossings.

In future work, our approximations will be tested on molecular systems by interfacing G-CT-MQC with various approaches to electronic-structure calculations, to investigate the possibility of improving those approximations similarly to strategies that are currently used in other contexts.

\section{Appendix: On the approximations in the spin-diabatic}

\section{representation}

In this Appendix we show how to derive the approximate expression given in Eq. (33) to determine the nuclear gradient of the phase of the spin-diabatic electronic coefficients.

Starting with the electronic equations 29 for $\dot{C}_{K}^{s d}(\mathbf{R}(t), t)$, and writing the coefficient in terms of its modulus and phase, we can isolate the evolution of the phase by equating all purely complex terms of the equation. Then, for $\left|C_{K}^{s d}(\mathbf{R}(t), t)\right| \neq 0$ we find

$$
\begin{aligned}
& \dot{\gamma}_{K}^{s d}(\mathbf{R}(t), t)=-\epsilon_{s d}^{(K)}(\mathbf{R}(t), t) \\
& -\sum_{J} \operatorname{Re}\left[\left[\hat{H}_{S O}(\mathbf{R}(t))\right]_{K J} e^{\frac{i}{\hbar}\left(\gamma_{J}^{s d}(\mathbf{R}(t), t)-\gamma_{K}^{s d}(\mathbf{R}(t), t)\right)}\right] \frac{\left|C_{J}^{s d}(\mathbf{R}(t), t)\right|}{\left|C_{K}^{s d}(\mathbf{R}(t), t)\right|} \\
& -\hbar \sum_{J} \sum_{\nu} \dot{\mathbf{R}}_{\nu}(t) \cdot \operatorname{Im}\left[\mathbf{d}_{\nu ; K J}(\mathbf{R}(t)) e^{\frac{i}{\hbar}\left(\gamma_{J}^{s d}(\mathbf{R}(t), t)-\gamma_{K}^{s d}(\mathbf{R}(t), t)\right)}\right] \frac{\left|C_{J}^{s d}(\mathbf{R}(t), t)\right|}{\left|C_{K}^{s d}(\mathbf{R}(t), t)\right|} .
\end{aligned}
$$


Last term, containing the NAC vectors, might be considered negligible if the NAC is suffi-

ciently localized in space if compared to the other two. Therefore, the gradient of $\gamma_{K}^{s d}(\mathbf{R}(t), t)$, that is

$$
\nabla_{\nu} \gamma_{K}^{s d}(\mathbf{R}(t), t)=\nabla_{\nu} \int^{t} d \tau \dot{\gamma}_{K}^{s d}(\mathbf{R}(\tau), \tau)
$$

can be written as

$$
\begin{aligned}
& \nabla_{\nu} \gamma_{K}^{s d}(\mathbf{R}(t), t)=\int^{t} d \tau\left(-\nabla_{\nu} \epsilon_{s d}^{(K)}(\mathbf{R}(\tau))\right) \\
& \quad-\int^{t} d \tau \nabla_{\nu} \sum_{J} \operatorname{Re}\left[\left[\hat{H}_{S O}(\mathbf{R}(\tau))\right]_{K J} e^{\frac{i}{\hbar}\left(\gamma_{J}^{s d}(\mathbf{R}(\tau), \tau)-\gamma_{K}^{s d}(\mathbf{R}(\tau), \tau)\right)}\right] \frac{\left|C_{J}^{s d}(\mathbf{R}(\tau), \tau)\right|}{\left|C_{K}^{s d}(\mathbf{R}(\tau), \tau)\right|}
\end{aligned}
$$

Computing the gradient in the second line would require calculations of nuclear gradients of the SOC, which we need to avoid envisaging the implementation of our algorithm with quantum-chemistry codes. Therefore, in the approximate expression for $\nabla_{\nu} \gamma_{K}^{s d}(\mathbf{R}(t), t)$ we only keep the first term that contains the gradients of the spin-diabatic energies.

\section{Acknowledgement}

This work is supported by a public grant from the "Laboratoire d'Excellence Physics Atoms Light Mater" (LabEx PALM) overseen by the French National Research Agency (ANR) as part of the "Investissements d'Avenir" program (reference: ANR-10- LABX-0039-PALM).

\section{Supporting Information Available}

The following files are available free of charge.

- TDPES.mp4: Supporting Information shows the evolution of the exact TDPES compared to its spin-diabatic and spin-adiabatic approximations based on G-CT-MQC. 


\section{References}

(1) Agostini, F.; Curchod, B. F. E.; Vuilleumier, R.; Tavernelli, I.; Gross, E. K. U. In Handbook of Materials Modeling; Andreoni, W., Yip, S., Eds.; Springer Netherlands, 2018; pp 1-47.

(2) Tavernelli, I.; Curchod, B. F. E.; Rothlisberger, U. Nonadiabatic molecular dynamics with solvent effects: A LR-TDDFT QM/MM study of ruthenium (II) tris (bipyridine) in water. Chem. Phys. 2011, 391, 101-109.

(3) Ando, H.; Iuchi, S.; Sato, H. Theoretical study on ultrafast intersystem crossing of chromium(III) acetylacetonate. Chem. Phys. Lett. 2012, 535, 177-181.

(4) Brahim, H.; Daniel, C. Structural and spectroscopic properties of Ir(III) complexes with phenylpyridine ligands: Absorption spectra without and with spin-orbit-coupling. Comput. Theo. Chem. 2014, 1040-1041, 219-229.

(5) Chergui, M. On the interplay between charge, spin and structural dynamics in transition metal complexes. Dalton Trans. 2012, 41, 13022-13029.

(6) van der Veen, R. M.; Cannizzo, A.; van Mourik, F.; Jr., A. V.; Chergui, M. Vibrational Relaxation and Intersystem Crossing of Binuclear Metal Complexes in Solution. J. Am. Chem. Soc. 2010, 133, 305-315.

(7) Cannizzo, A.; Blanco-Rodríguez, A. M.; Nahha, A. E.; Šebera, J.; Záliš, S.; Jr., A. V.; Chergui, M. Femtosecond Fluorescence and Intersystem Crossing in Rhenium(I) Carbonyl-Bipyridine Complexes. J. Am. Chem. Soc. 2008, 130, 8967-8974.

(8) Talotta, F.; Heully, J.-L.; Alary, F.; Dixon, I. M.; González, L.; Boggio-Pasqua, M. Linkage Photoisomerization Mechanism in a Photochromic Ruthenium Nitrosyl Complex: New Insights from an MS-CASPT2 Study. J. Chem. Theory Comput. 2017, 13, 6120-6130. 
(9) Atkins, A. J.; Talotta, F.; Freitag, L.; Boggio-Pasqua, M.; González, L. Assessing Excited State Energy Gaps with Time-Dependent Density Functional Theory on Ru(II) Complexes. J. Chem. Theory Comput. 2017, 13, 4123-4145.

(10) Garcáa, J. S.; Talotta, F.; Alary, F.; Dixon, I. M.; Heully, J.-L.; Boggio-Pasqua, M. A Theoretical Study of the N to O Linkage Photoisomerization Efficiency in a Series of Ruthenium Mononitrosyl Complexes. Molecules 2017, 22, 1667.

(11) Talotta, F.; Boggio-Pasqua, M.; González, L. Early relaxation dynamics in the photoswitchable trans- $\left[\mathrm{RuCl}(\mathrm{NO})(\mathrm{py})_{4}\right]^{2+}$. Chem.: Eur. J. (accepted) 2020,

(12) Parker, D. S. N.; Minns, R. S.; Penfold, T. J.; Worth, G. A.; Fielding, H. H. Ultrafast dynamics of the $\mathrm{S}_{1}$ excited state of benzene. Chem. Phys. Lett. 2009, 469, 43-47.

(13) Han, B.; Zheng, Y. Nonadiabatic Quantum Dynamics in $\mathrm{O}\left({ }^{3} \mathrm{P}\right)+\mathrm{H}_{2} \rightarrow \mathrm{OH}+\mathrm{H}$ : A Revisited Study. J. Comput. Chem. 2011, 32, 3520-3525.

(14) Mai, S.; Marquetand, P.; González, L. Non-adiabatic and intersystem crossing dynamics in $\mathrm{SO}_{2}$. II. The role of triplet states in the bound state dynamics studied by surfacehopping simulations. J. Chem. Phys. 2014, 140, 204302.

(15) Rajak, K.; Maiti, B. Communications: Direct dynamics study of the $\mathrm{O}\left({ }^{3} \mathrm{P}\right)+\mathrm{C}_{2} \mathrm{H}_{2}$ reaction: Contribution from spin nonconserving route. J. Chem. Phys. 2010, 133, 011101.

(16) Fu, B.; Han, Y.-C.; Bowman, J. M.; Angelucci, L.; Balucani, N.; Leonori, F.; Casavecchia, P. Intersystem crossing and dynamics in $\mathrm{O}\left({ }^{3} \mathrm{P}\right)+\mathrm{C}_{2} \mathrm{H}_{4}$ multichannel reaction: Experiment validates theory. Proc. Natl. Acad. Sci. 2012, 109, 9733-9738.

(17) Minns, R. S.; Parker, D. S. N.; Penfold, T. J.; Worth, G. A.; Fielding, H. H. Competing ultrafast intersystem crossing and internal conversion in the "channel 3" region of benzene. Phys. Chem. Chem. Phys. 2010, 12, 15607-15615. 
(18) Li, H.; Kamasah, A.; Matsika, S.; Suits, A. G. Intersystem crossing in the exit channel. Nature Chem. 2019, 11, 123-128.

(19) Martínez-Fernández, L.; Corral, I.; Granucci, G.; Persico, M. Competing ultrafast intersystem crossing and internal conversion: a time resolved picture for the deactivation of 6-thioguanine. Chem. Sci. 2014, 5, 1336-1347.

(20) Richter, M.; Marquetand, P.; González-Vázquez, J.; Sola, I.; González, L. Femtosecond Intersystem Crossing in the DNA Nucleobase Cytosine. J. Phys. Chem. Lett. 2012, 3, $3090-3095$.

(21) Abedi, A.; Maitra, N. T.; Gross, E. K. U. Exact factorization of the time-dependent electron-nuclear wave function. Phys. Rev. Lett. 2010, 105, 123002.

(22) Abedi, A.; Maitra, N. T.; Gross, E. K. U. Correlated electron-nuclear dynamics: Exact factorization of the molecular wave-function. J. Chem. Phys. 2012, 13\%, 22A530.

(23) Agostini, F.; Curchod, B. F. E. Different flavors of nonadiabatic molecular dynamics. WIREs Comput. Mol. Sci. 2019, 9, e1417.

(24) Min, S. K.; Agostini, F.; Gross, E. K. U. Coupled-trajectory quantum-classical approach to electronic decoherence in nonadiabatic processes. Phys. Rev. Lett. 2015, 115, 073001.

(25) Agostini, F.; Min, S. K.; Abedi, A.; Gross, E. K. U. Quantum-classical non-adiabatic dynamics: Coupled- vs. independent-trajectory methods. J. Chem. Theory Comput. 2016, 12, 2127-2143.

(26) Min, S. K.; Agostini, F.; Tavernelli, I.; Gross, E. K. U. Ab Initio Nonadiabatic Dynamics with Coupled Trajectories: A Rigorous Approach to Quantum (De)Coherence. J. Phys. Chem. Lett. 2017, 8, 3048-3055.

(27) Curchod, B. F. E.; Agostini, F.; Tavernelli, I. CT-MQC - A Coupled-Trajectory Mixed 
Quantum/Classical method including nonadiabatic quantum coherence effects. Euro. Phys. J. B 2018, 91, 168.

(28) Gossel, G.; Agostini, F.; Maitra, N. T. Coupled-Trajectory Mixed Quantum-Classical Algorithm: A Deconstruction. J. Chem. Theory Comput. 2018, 14, 4513-4529.

(29) Ha, J.-K.; Lee, I. S.; Min, S. K. Surface Hopping Dynamics Beyond Nonadiabatic Couplings for Quantum Coherence. J. Phys. Chem. Lett. 2018, 9, 1097-1104.

(30) Talotta, F.; Morisset, S.; Rougeau, N.; Lauvergnat, D.; Agostini, F. Spin-Orbit Interactions in Ultrafast Molecular Processes. Phys. Rev. Lett. 2020, 124, 033001.

(31) Curchod, B. F. E.; Rauer, C.; Marquetand, P.; González, L.; Martínez, T. J. Communication: GAIMS - Generalized Ab Initio Multiple Spawning for both internal conversion and intersystem crossing processes. J. Chem. Phys. 2016, 144, 101102.

(32) de Carvalho, F. F.; Tavernelli, I. Nonadiabatic dynamics with intersystem crossings: A time-dependent density functional theory implementation. J. Chem. Phys. 2015, 143, 224105.

(33) Cui, G.; Thiel, W. Generalized trajectory surface-hopping method for internal conversion and intersystem crossing. J. Chem. Phys. 2014, 141, 124101.

(34) Richter, M.; Marquetand, P.; González-Vázquez, J.; Solab, I.; González, L. SHARC: ab Initio Molecular Dynamics with Surface Hopping in the Adiabatic Representation Including Arbitrary Couplings. J. Chem. Theory Comput. 2011, 7, 1253-1258.

(35) Hu, W.; Lendvay, G.; Maiti, B.; Schatz, G. C. Trajectory Surface Hopping Study of the $\mathrm{O}\left({ }^{3} \mathrm{P}\right)+$ Ethylene Reaction Dynamics. J. Phys. Chem. A 2008, 112, 2093-2103.

(36) Valero, R.; Truhlar, D. G. A Diabatic Representation Including Both Valence Nonadiabatic Interactions and Spin-Orbit Effects for Reaction Dynamics. J. Phys. Chem. A 2007, 111, 8536 . 
(37) Marquetand, P.; Richter, M.; González-Vázquez, J.; Sola, I.; González, L. Nonadiabatic ab initio molecular dynamics including spin-orbit coupling and laser fields. Faraday Discuss. 2011, 153, 261-273.

(38) Mai, S.; Marquetand, P.; González, L. A General Method to Describe Intersystem Crossing Dynamics in Trajectory Surface Hopping. Int. J. Quant. Chem. 2015, 115, $1215-1231$.

(39) Granucci, G.; Persico, M.; Spighi, G. Surface hopping trajectory simulations with spinorbit and dynamical couplings. J. Chem. Phys. 2012, 137, 22 A501.

(40) Fedorov, D. A.; Pruitt, S. R.; Keipert, K.; Gordon, M. S.; Varganov, S. A. Ab Initio Multiple Spawning Method for Intersystem Crossing Dynamics: Spin-Forbidden Transitions between ${ }^{3} \mathrm{~B}^{1}$ and ${ }^{1} \mathrm{~A}^{1}$ States of $\mathrm{GeH}_{2}$. J. Phys. Chem. A. 2016, 120, 2911-2919.

(41) Fedorov, D. A.; Lykhin, A. O.; Varganov, S. A. Predicting Intersystem Crossing Rates with AIMS-DFT Molecular Dynamics. J. Phys. Chem. A. 2018, 122, 3480-3488.

(42) Penfold, T. J.; Gindensperger, E.; Daniel, C.; Marian, C. M. Spin-Vibronic Mechanism for Intersystem Crossing. Chem. Rev. 2018, 118, 6975-7025.

(43) Capano, G.; Chergui, M.; Rothlisberger, U.; Tavernelli, I.; Penfold, T. J. A Quantum Dynamics Study of the Ultrafast Relaxation in a Prototypical $\mathrm{Cu}(\mathrm{I})-$ Phenanthroline. J. Phys. Chem. A 2014, 118, 9861-9869.

(44) van Lenthe, E.; Baerends, E. J.; Snijders, J. G. Relativistic regular two-component Hamiltonians. J. Chem. Phys. 1993, 99, 4597.

(45) Marian, C. M. Spin-orbit coupling and intersystem crossing in molecules. WIREs Comput. Mol. Sci. 2012, 2, 187-203. 
(46) Alonso, J. L.; Clemente-Gallardo, J.; Echeniche-Robba, P.; Jover-Galtier, J. A. Comment on "Correlated electron-nuclear dynamics: Exact factorization of the molecular wave-function". J. Chem. Phys. 2013, 139, 087101.

(47) Abedi, A.; Maitra, N. T.; Gross, E. K. U. Reply to Comment on "Correlated electronnuclear dynamics: Exact factorization of the molecular wave-function". J. Chem. Phys. 2013, 139, 087102 .

(48) Agostini, F.; Tavernelli, I.; Ciccotti, G. Nuclear Quantum Effects in Electronic (Non)Adiabatic Dynamics. Euro. Phys. J. B 2018, 91, 139.

(49) Requist, R.; Tandetzky, F.; Gross, E. K. U. Molecular geometric phase from the exact electron-nuclear factorization. Phys. Rev. A 2016, 93, 042108.

(50) Requist, R.; Proetto, C. R.; Gross, E. K. U. Asymptotic analysis of the Berry curvature in the E $\otimes$ e Jahn-Teller model. Phys. Rev. A 2017, 96, 062503.

(51) Curchod, B. F. E.; Agostini, F. On the Dynamics through a Conical Intersection. J. Phys. Chem. Lett. 2017, 8, 831-837.

(52) Agostini, F.; Curchod, B. F. E. When the Exact Factorization Meets Conical Intersections... Euro. Phys. J. B 2018, 91, 141.

(53) Abedi, A.; Agostini, F.; Suzuki, Y.; Gross, E. K. U. Dynamical steps that bridge piecewise adiabatic shapes in the exact time-dependent potential energy surface. Phys. Rev. Lett 2013, 110, 263001.

(54) Agostini, F.; Abedi, A.; Suzuki, Y.; Gross, E. K. U. Mixed quantum-classical dynamics on the exact time-dependent potential energy surfaces: A novel perspective on nonadiabatic processes. Mol. Phys. 2013, 111, 3625-3640.

(55) Min, S. K.; Abedi, A.; Kim, K. S.; Gross, E. K. U. Is the molecular Berry phase an artefact of the Born-Oppenheimer approximation? Phys. Rev. Lett. 2014, 113, 263004. 
(56) Agostini, F.; Abedi, A.; Suzuki, Y.; Min, S. K.; Maitra, N. T.; Gross, E. K. U. The exact forces on classical nuclei in non-adiabatic charge transfer. J. Chem. Phys. 2015, 142, 084303.

(57) Curchod, B. F. E.; Agostini, F.; Gross, E. K. U. An exact factorization perspective on quantum interferences in nonadiabatic dynamics. J. Chem. Phys. 2016, 145, 034103.

(58) Lacombe, L.; Hoffmann, N. M.; Maitra, N. T. Exact Potential Energy Surface for Molecules in Cavities. Phys. Rev. Lett. 2019, 123, 083201.

(59) Abedi, A.; Agostini, F.; Gross, E. K. U. Mixed quantum-classical dynamics from the exact decomposition of electron-nuclear motion. Europhys. Lett. 2014, 106, 33001.

(60) Agostini, F.; Abedi, A.; Gross, E. K. U. Classical nuclear motion coupled to electronic non-adiabatic transitions. J. Chem. Phys. 2014, 141, 214101.

(61) Agostini, F.; Min, S. K.; Gross, E. K. U. Semiclassical analysis of the electron-nuclear coupling in electronic non-adiabatic processes. Ann. Phys. 2015, 527, 546-555.

(62) Gossel, G. H.; Lacombe, L.; Maitra, N. T. On the Numerical Solution of the Exact Factorization Equations. J. Chem. Phys. 2019, 150, 154112.

(63) Granucci, G.; Persico, M. Gradients for Configuration Interaction Energies with SpinOrbit Coupling in a Semiempirical Framework. J. Comput. Chem. 2011, 32, 2690-2696.

(64) Agostini, F. An exact-factorization perspective on quantum-classical approaches to excited-state dynamics. Euro. Phys. J. B 2018, 91, 143.

(65) Scherrer, A.; Agostini, F.; Sebastiani, D.; Gross, E. K. U.; Vuilleumier, R. Nuclear velocity perturbation theory for vibrational circular dichroism: An approach based on the exact factorization of the electron-nuclear wave function. J. Chem. Phys. 2015, $143,074106$. 
(66) Eich, F. G.; Agostini, F. The adiabatic limit of the exact factorization of the electronnuclear wave function. J. Chem. Phys. 2016, 145, 054110.

(67) Scherrer, A.; Agostini, F.; Sebastiani, D.; Gross, E. K. U.; Vuilleumier, R. On the mass of atoms in molecules: Beyond the Born-Oppenheimer approximation. Phys. Rev. X 2017, \%, 031035.

(68) Böckmann, M.; Klessinger, M.; Zerner, M. C. Spin-Orbit Coupling in Organic Molecules:? A Semiempirical Configuration Interaction Approach toward Triplet State Reactivity. J. Phys. Chem. 1996, 100, 10570-10579.

(69) Tal-Ezer, H.; Kosloff, R. An accurate and efficient scheme for propagating the time dependent Schrödinger equation. J. Chem. Phys. 1984, 81, 3967.

(70) Leforestier, C.; Bisseling, R. H.; Cerjan, C.; Feit, M. D.; Friesner, R.; Guldberg, A.; Hammerich, A.; Jolicard, G.; Karrlein, W.; Meyer, H.-D.; Lipkin, N.; Roncero, O.; Kosloff, R. A comparison of different propagation schemes for the time dependent Schrödinger equation. J. Comput. Phys. 1991, 94, 59-80.

(71) Lauvergnat, D. ElVibRot: Quantum dynamics code. https://github.com/lauvergn/ ElVibRot-TnumTana.

(72) Muckerman, J. Some useful discrete variable representations for problems in timedependent and time-independent quantum mechanics. Chem. Phys. Lett. 1990, 173, 200 .

(73) Agostini, F.; Min, S. K.; Tavernelli, I.; Gossel, G. H. 2018; CTMQC.

(74) Fu, B.; Shepler, B. C.; Bowman, J. M. Three-State Trajecory Surface Hopping Studies of the Photodissociation Dynamics of Formaldehyde on ab Initio Potential Energy Surfaces. J. Am. Chem. Soc. 2011, 133, 7957-7968. 
Graphical TOC Entry

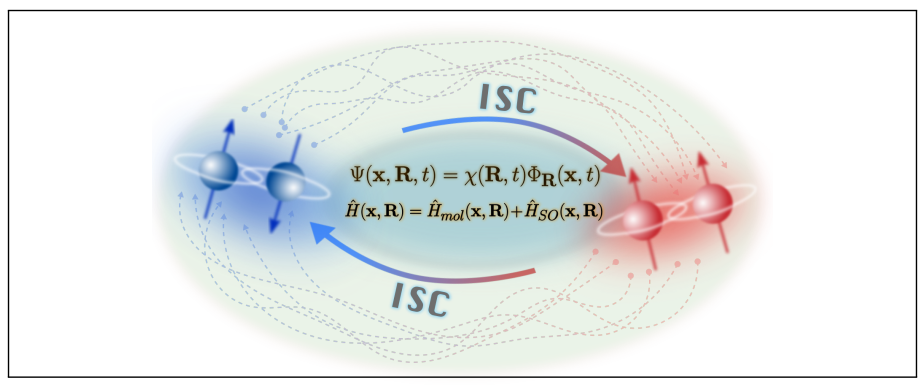

